\title{
CONJUGACY OF REAL DIFFEOMORPHISMS. A SURVEY
}

\author{
A. G. O'FARRELL AND M. ROGINSKAYA \\ Dedicated to V. P. Havin \\ on the occasion of his 75th birthday
}

\begin{abstract}
Given a group $G$, the conjugacy problem in $G$ is the problem of giving an effective procedure for determining whether or not two given elements $f, g \in G$ are conjugate, i. e., whether there exists $h \in G$ with $f h=h g$. This paper is about the conjugacy problem in the group $\operatorname{Diffeo}(I)$ of all diffeomorphisms of an interval $I \subset \mathbb{R}$.

There is much classical work on the subject, solving the conjugacy problem for special classes of maps. Unfortunately, it is also true that many results and arguments known to the experts are difficult to find in the literature, or simply absent. We try to repair these lacunae, by giving a systematic review, and we also include new results about the conjugacy classification in the general case.
\end{abstract}

\section{§1. INFORMAL INTRODUCTION}

1.1. Objective. We are going to work with diffeomorphisms defined just on various intervals (open, closed, or half-open, bounded or unbounded). Let Diffeo( $I$ ) denote the group of (infinitely differentiable) diffeomorphisms of the interval $I \subset \mathbb{R}$, under the operation of composition. We denote the (normal) subgroup of orientation-preserving diffeomorphisms of the interval by $\operatorname{Diffeo}^{+}(I)$. If an endpoint $c$ belongs to $I$, then statements about derivatives at $c$ should be interpreted as referring to one-sided derivatives.

Our objective is to classify the conjugacy classes, i.e., to determine when two given maps $f$ and $g$ are conjugate in $\operatorname{Diffeo}(I)$.

The reason this problem is important is that conjugate elements correspond to one another under a "change of variables". For most applications, a change of variables will not alter anything essential, so only the conjugacy class of an element is significant. From the viewpoint of group theorists, it is also usual to regard only the conjugacy classes as having "real" meaning in a group.

Throughout the paper, we will use the term smooth to mean infinitely differentiable. There is a good deal of valuable and delicate work on conjugacy problems for functions that are merely $C^{k}$, but we will not delve into this (apart from an occasional remark), in order to keep the discussion within bounds.

Apart from its intrinsic interest, the conjugacy problem has applications to the holonomy theory of codimension-one foliations. Mather established a connection between the homotopy of Haefliger's classifying space for foliations and the cohomology of the group $G$ of compactly supported diffeomorphisms of the line [15, 16. Mather also used a conjugacy classification of a subset of the group $G$ in order to establish that $G$ is perfect.

2010 Mathematics Subject Classification. Primary 37E05, 20 E45.

Key words and phrases. Diffeomorphism group, conjugacy, real line, orientation.

Supported by Grant SFI RFP05/MAT0003 and the ESF Network HCAA. 
It follows from a result of Epstein that $G$ is simple. Our own study of the conjugacy problem arose independently from our interest in reversible maps (maps conjugate to their own inverses) 24.

We should make it clear that we are not here discussing an example of the classical Dehn conjugacy problem of combinatorial group theory. The group $\operatorname{Diffeo}^{+}(I)$ is not countably presented. It has the cardinality of the continuum. Its family of conjugacy classes also has the cardinality of the continuum. To classify conjugacy classes is a matter of identifying suitable conjugacy invariants which separate the classes. To be of practical use, the invariants should be reasonably "computable", in some sense, but the sense has to be more lax than standard Turing-machine computability. For a start, we assume that we have available a "real computer", that can do real arithmetic and decide the equality of two suitably specified real numbers. We include as suitable specifications things such as the value of an integral of a suitably explicit function, and the limit of a suitably explicit sequence. In practice, the kind of problem one wishes to solve is this: given a prescription for two diffeomorphisms $f$ and $g$, sufficiently explicit that we may compute the images of any suitably specified point, decide whether or not they are conjugate. This may seem quite modest (especially as we have not made explicit what is meant by "suitably explicit") but, as we shall see, it is rather too much to hope for. A less demanding task would be to come up with a procedure that will confirm that two nonconjugate diffeomorphisms are in fact nonconjugate, but may go on forever if presented with two conjugates. Even this is too much, except in special cases. What one can do is provide a collection of classifying invariants that provide a significant conceptual simplification of the conjugacy problem.

The conjugacy problem in $\operatorname{Diffeo}(I)$ may be reduced to the corresponding problem in the subgroup $\operatorname{Diffeo}^{+}(I)$ of orientation-preserving maps of $I$. This recent result is described in 89, A crucial case of the latter problem is the special case in which the diffeomorphisms $f, g \in \operatorname{Diffeo}^{+}(I)$ are fixed-point-free on the interior $J$ of $I$. The problem is trivial if $I$ is open (Proposition 2.1). A new result (Theorem 2.5) provides an effective way to approach it when $I$ is half-open. We establish that it suffices to search for a conjugacy $h$ among the solutions of a first-order ordinary differential equation. This also helps with the case of compact $I$. For special ("flowable") diffeomorphisms of a compact $I$, the conjugacy classification can be achieved using the so-called "functional moduli", similar in character to the Écalle-Voronin moduli for the conjugacy classification of biholomorphic germs [31. In the general case, this cannot be done.

There has been much work on this problem. Important steps in the story we describe below are the work of Sternberg, Takens, Sergeraert, Robbin, Mather, Young, and Kopell, among others. There is a useful summary survey of progress up to 1995 by Ahern and Rosay [2]. See also references [18, 25, 27, 28, 30]; 13, Chapter 8]; [12, Chapter 2]; [1, 3, 8, 19, 29, 33, 34]]. There are some parallels with the conjugacy problem for complex analytic germs, for which see [5].

1.2. Notation. We shall use Diffeo as an abbreviation for Diffeo $(I)$, and $\mathrm{Diffeo}^{+}$for Diffeo $^{+}(I)$, whenever there is no danger of confusion.

For $f \in \operatorname{Diffeo}(I)$, we denote the set of fixed points of $f$ by fix $(f)$.

We use the symbol $f^{\circ n}$ for the $n$th iterate of $f$ (i.e., the $n$th power in the group Diffeo $(I))$. We also use it for negative $n=-m$ to denote the $m$ th iterate of the inverse function $f^{\circ-1}$. The notation $f^{\circ 0}$ denotes the identity map $\mathbb{1}$.

We use similar notation for compositional powers and inverses in the group $F$ of formally invertible formal power series (with real coefficients) in the indeterminate $X$. The identity $X+0 X^{2}+0 X^{3}+\cdots$ is denoted simply by $X$. 
We denote $g^{h}=h^{\circ-1} \circ g \circ h$, whenever $g, h \in \operatorname{Diffeo}(I)$. We say that $h$ conjugates $f$ to $g$ if $f=g^{h}$.

We use the notation $\operatorname{deg} f$ for the degree of the diffeomorphism $f \in \operatorname{Diffeo}(I)(= \pm 1$, depending on whether or not $f$ preserves the order on $I$ ). Thus

$$
\operatorname{Diffeo}^{+}(I)=\{f \in \operatorname{Diffeo}(I): \operatorname{deg} f=+1\} .
$$

Given a closed set $E \subset I$, we set

$$
\text { Diffeo }_{E}^{+}=\operatorname{Diffeo}_{E}^{+}(I)=\left\{f \in \operatorname{Diffeo}^{+}(I): f(x)=x, \forall x \in E\right\},
$$

the subgroup of those direction-preserving maps that fix each point of $E$.

We denote the map $x \mapsto-x$ on $\mathbb{R}$ by - .

When we come to discuss conjugation for elements having complicated fixed-point sets, we will need notation for the available conjugacies on particular subintervals. So we make a definition.

Definition. Let $f, g \in \operatorname{Diffeo}^{+}(I)$. Given an open interval $J \subset I$ that is mapped onto itself by $f$ and by $g$, we say that a map $\phi \in \operatorname{Diffeo}(\operatorname{clos}(J))$ is a $J$-conjugation from $f$ to $g$ if $f^{\phi}=g$ on $\operatorname{clos}(J)$. We denote the set of all $J$-conjugations from $f$ to $g$ by $\operatorname{Conj}(f, g ; J)$, or just $\operatorname{Conj}(J)$, if the context is clear.

1.3. Remarks about topological conjugacy. A necessary condition for the conjugacy of two elements $f, g \in \operatorname{Diffeo}(I)$ is that they be topologically conjugate, i.e., conjugate in the homeomorphism group $\operatorname{Homeo}(I)$.

The homeomorphism problem is strictly easier than the diffeomorphism problem, because it is included as part of it: (1) One can show that each conjugacy class of homeomorphisms has an element that is a diffeomorphism. (2) Thus, if one knows how to classify diffeomorphisms up to topological conjugacy, then one knows how to classify homeomorphisms also. (3) The topological conjugacy classification is coarser than the diffeomorphic one.

As we shall now explain, the topological conjugacy problem is already intractable, in computational terms, so it follows that the same is true for smooth conjugacy.

Let us consider the case $I=\mathbb{R}$.

The conjugacy problem in the homeomorphism group $\operatorname{Homeo}(\mathbb{R})$ has a classical solution in terms of a "symbol" invariant. This goes back, essentially, to Sternberg [28], who in 1957 described the conjugacy classes in the group of germs of homeomorphisms of neighborhoods of a point on the line. For an exposition of the classification in $\operatorname{Homeo}(\mathbb{R})$, see [21]. Mere topological conjugacy of two direction-preserving diffeomorphisms $f$ and $g$ is determined by the existence of a homeomorphism of $\mathbb{R}$ mapping fix $(f)$ onto fix $(g)$ and coincidence of the "pattern of signs" of $f(x)-x$ and $g(x)-x$ off the fixed-point sets. (The pattern of signs of $f(x)-x$ is called the "signature" of $f$.)

Suppose $f=g^{h}$. Then $h$ carries $F_{1}=\operatorname{fix}(f)$ onto $F_{2}=\operatorname{fix}(g)$, so the pairs $(\mathbb{R}, \operatorname{fix}(f))$ and $(\mathbb{R}$, fix $(g))$ are homeomorphic. An order-isomorphism between two partially ordered sets is an order-preserving bijection. Two partially ordered sets are order-isomorphic if there exists an order-isomorphism between them. Order-isomorphism is an equivalence relation on the family of partially ordered sets, and the equivalence classes are called order classes. The homeomorphism class of a pair $(\mathbb{R}, F)$ (with $F$ closed) is determined by the order class of $F$ (with the usual total order inherited from $\mathbb{R}$ ). Every closed subset of $\mathbb{R}$ is the fixed-point set of some homeomorphism (and even of some diffeomorphism), so the set fix $(f)$ may be quite general. Thus there are two obstacles to finding an algorithmic solution to the topological conjugacy problem.

(1) The problem of determining whether two closed subsets of $\mathbb{R}$ are order-equivalent does not appear to be amenable to an algorithmic solution. For subsets of a simple 
structure it may be resolved by noting that an order-isomorphism will induce a bijection of the derived set, the second derived set, and so on through the ordinals, a bijection of the relative complements of each of these, a bijection of the condensation set, a bijection of each interval subset, and of the ends of such intervals, and of derived sets of ends, etc. But a general algorithm is another matter.

(2) There may be a large collection of order-isomorphisms between fix $(f)$ and fix $(g)$, and we then need some systematic way to check for the existence of one order-isomorphism that gives a coincidence of signatures on the corresponding complementary intervals.

Returning to the problem of $C^{\infty}$ conjugacy, we have additional complications, as the following observations indicate.

1.4. Smooth conjugacy of pairs. Suppose $f, g, h \in \operatorname{Diffeo}(I)$ and $f=g^{h}$. Then $h$ carries $F_{1}=\operatorname{fix}(f)$ onto $F_{2}=\operatorname{fix}(g)$, so the pairs $(\mathbb{R}, \operatorname{fix}(f))$ and $(\mathbb{R}, f i x(g))$ are diffeomorphic. This necessary condition is more complex to check than the corresponding topological condition. To determine whether two homeomorphic pairs belong to the same diffeomorphism class, it is necessary to search among all the order-isomorphisms of the $F_{i}$ 's for one having a diffeomorphic extension. The existence of a diffeomorphic extension may be checked using a theorem of Whitney. Whitney's condition [32] for the existence of a $C^{\infty}$ extension of a function $h$ from $F_{1}$ to $\mathbb{R}$ may be stated as follows: For each $k \in \mathbb{N}$, the $k$ th Newton divided difference of $h$ is uniformly continuous on bounded subsets of

$$
\left\{\left(x_{1}, \ldots, x_{k+1}\right) \in F_{1} \times \cdots \times F_{1}: x_{i} \neq x_{j}, \forall i \neq j\right\}
$$

(i. e., extends continuously to the full product $F_{1} \times \cdots \times F_{1}$ ). In fact, an order-isomorphism has a diffeomorphic extension if and only if it has an infinitely differentiable extension, and the (uniquely determined) first derivative of such an extension is nonzero at each accumulation point.

1.5. Orbits, multipliers, and Taylor series. The (two-sided) orbit of a point $a \in \mathbb{R}$ under a diffeomorphism $f$ is the set $\left\{f^{\circ n}(a): n \in \mathbb{Z}\right\}$ of all forward and backward images of $a$ under the action of $f$. If $f, g, h \in \operatorname{Diffeo}(\mathbb{R})$ and $f=g^{h}$, then for each $a \in \mathbb{R}$, the map $h$ carries the orbit $O_{1}$ of $a$ under $f$ onto a corresponding orbit $O_{2}$ under $g$, so the pairs $\left(\mathbb{R}, O_{1}\right)$ and $\left(\mathbb{R}, O_{2}\right)$ are diffeomorphic. An implication is that these pairs are equivalent under locally bi-Lipschitzian maps. Thus, for instance, one sees (by estimating the number of points in orbits in intervals of comparable length) that the maps defined by

$$
f(x)=x+\exp \left(-1 / x^{2}\right)
$$

and

$$
g(x)=x+\exp \left(-2 / x^{2}\right)
$$

are not conjugate, although they have identical signatures.

It is straightforward (using Whitney's result) to check whether two orbit pairs $\left(\mathbb{R}, O_{1}\right)$ and $\left(\mathbb{R}, O_{2}\right)$ are diffeomorphic, but a difficulty is that one must check that for each orbit of $f$ there exists some orbit of $g$ that gives a diffeomorphic pair. This is not a constructive condition, as it stands.

Further, if $f=g^{h}$, then for each $a \in \operatorname{fix}(f)$, letting $b=h(a)$, we have

$$
g^{\prime}(b)=f^{\prime}(a)
$$

i.e., $f$ and $g$ have the same "multipliers" at corresponding points. This necessary condition actually follows from the previous one about orbits when $a$ is a boundary point of fix $(f)$, but it is easier to check when it fails. It is trivial at accumulation points of fix $(f)$. 
There is a more elaborate necessary condition involving higher derivatives, best expressed in terms of Taylor series: Let $T_{a} f$ denote the truncated Taylor series of $f$ about $a$ :

$$
T_{a} f=\sum_{n=1}^{\infty} \frac{f^{(n)}(a)}{n !} X^{n}
$$

(regarded as a formal power series in an indeterminate $X$ ). One then has

$$
T_{a} f=\left(T_{a} h\right)^{\circ-1} \circ\left(T_{b} g\right) \circ\left(T_{a} h\right),
$$

where $\circ$ denotes the formal composition, and $p^{\circ-1}$ denotes the formal compositional inverse. This condition is weaker than the one about orbits, since the Taylor series at a point is determined by the values of the function at any sequence tending to the point.

However, there is a straightforward algorithm for checking whether or not two formal power series are formally conjugate. In fact, each series is conjugate to one of $\lambda X(\lambda \in \mathbb{R})$, or one of $\pm X \pm X^{p+1}+\alpha X^{2 p+1}$, and in each case the correct class can be determined by a terminating computation. This fact is well known (cf. [2, p. 546]; 6, 11, 22]) and is routine to check. The main point to note is that the group of invertible formal power series (with its product topology) is topologically generated by the maps $x \mapsto \lambda X(\lambda \neq 0)$ and $x \mapsto x+\alpha x^{p+1}(p \in \mathbb{N})$ [22, Lemma 1, p. 5].

For example:

(1) $3 X+X^{2}$ is conjugate to $3 X+2 X^{2}$, and to any other series that begins with $3 X$, but is not conjugate to any series that begins with $2 X$.

(2) $X+X^{2}+X^{3}$ is conjugate to $X+2 X^{2}+4 X^{3}+8 X^{4}+\cdots$, but not to any series beginning with $X+3 X^{2}+6 X^{3}$ or $X+2 X^{3}$.

(3) Each series beginning with $X+X^{4}+2 X^{7}$ is conjugate to each series beginning with $X+5 X^{4}+50 X^{7}$.

We will see below that there is more to conjugacy than the diffeomorphism of pairs, correspondence of signatures, and the orbit conditions, but that the problem can nevertheless be reduced to manageable proportions, provided one does not try to do the impossible.

1.6. Centralizers. Typically, if $f$ and $g$ are conjugate diffeomorphisms, then the family $\Phi$ of diffeomorphisms $\phi$ such that $f=\phi^{\circ-1} \circ g \circ \phi$ has more than one element. In fact $\Phi$ is a left coset of the centralizer $C_{f}$ of $f$ (and a right coset of $C_{g}$ ). For this reason, it is important for us to understand the structure of these centralizers. The problem of describing $C_{f}$ is a special conjugacy problem: Which maps conjugate $f$ to itself?

Historically, there has been a good deal more work on the problem of centralizers than on the general conjugacy problem.

There may be a great many conjugacies between two given conjugate diffeomorphisms. In the open-interval case, the centralizer of a fixed-point-free diffeomorphism is very large and is not Abelian.

Kopell [10] showed that when $I$ has one of its endpoints as a member, then the centralizer of an $f$ that is fixed-point-free on the interior of $I$ must be quite small; it is a subgroup of a one-parameter Abelian group, and it may consist just of the iterates of $f$. An example was given by Sergeraert [27]; probably this behavior is "generic". Kopell [10] showed that it is generic for maps that fix only the two endpoints of $I$. These phenomena tell us that in many cases the search for a conjugating map $h$ from $f$ to $g$ may be confined to a 1-parameter search space. Our main new theorem gives a specific way to locate this search space, in the case of a half-open interval. (See Subsection 2.11 and \$6] ) 
1.7. Outline. The paper is organized as follows.

First, we consider various special cases of the full conjugacy problem, and related simpler problems, and then we use these cases and problems as building blocks in constructing a solution to the full problem.

The results are summarized formally in $\$ 2$. The remaining sections provide proofs, elaboration, and examples.

In less formal terms, we proceed as follows.

We start with the simple and classical case of fixed-point-free maps of an open interval, where there is just one conjugacy class of diffeomorphisms. (The details are in $\sqrt{3}$.)

Then we study conjugacy in half-open intervals, starting with diffeomorphisms of the interval $[0,+\infty)$ that fix only 0 . First, we review the classical results based on normal forms that exist when the diffeomorphism is not tangent to the identity to infinite order. In these special cases the conditions simplify. If the multiplier at 0 is not 1 (i.e., 0 is a hyperbolic fixed point), then Sternberg [28] identified the multiplier as the sole conjugacy invariant. If the multiplier is 1 , but $f$ is not "infinitesimally tangent to the identity" (i.e., $T_{0} f \neq X$ - we find it less of a mouthful to express this condition as " $f-x$ is not flat at $p$ "), then Takens [30] identified the conjugacy class of the Taylor series $T_{0} f$ (in the group of formally-invertible power series) as the sole conjugacy invariant. We show that the general problem cannot be tackled using normal forms. We identify an infinite product condition that is necessary for conjugacy. We then base our approach to characterizing conjugacy on a certain differential equation that may be formulated when the product condition holds. (The details are in $\$ \$ 4$, 5] and 6.)

Next, we study conjugacy in $\operatorname{Diffeo}^{+}(I)$, for closed bounded intervals $I$, for maps that are fixed-point-free on the interior $J$ of $I$. In the "Axiom A" case, in which both fixed points are hyperbolic, Robbin characterized conjugacy in terms of the multipliers and a "modulus" (a smooth function on $(0,+\infty)$; details below). The results of Young [34] relate to other cases in which $f-x$ is not flat at either end of $I$, particularly the "saddlenode" case, in which $T_{0} f-X$ is zero $\bmod X^{2}$, but not zero $\bmod X^{3}$, for both endpoints $p$. He used so-called "formal multipliers" (certain diffeomorphisms from $J$ to $(0,+\infty)$ ) to construct a substitute for the Robbin modulus, which, when taken together with the conjugacy classes of the Taylor series at the ends, characterizes conjugacy classes. There is a more general treatment of functional moduli ideas in unpublished work of Mather [15. We give a necessary and sufficient condition for conjugacy that builds on the result for half-open intervals. We also review functional moduli in the special Mather case, and a useful new necessary condition expressed in terms of the "shape" of a graph associated to the pair of maps $(f, g)$. (The details are in $\$ 7$.)

Then we move on to general direction-preserving diffeomorphisms, on any interval $I$, with possibly complicated fixed-point sets. We take this in two stages.

(1) We reduce the conjugacy problem in $\operatorname{Diffeo}(I)^{+}$to one in $\operatorname{Diffeo}_{\mathrm{bdy} E}^{+}(I)$, for a fixed closed E. (The details are in Subsection 2.12.)

(2) We address the conjugacy problem in $\operatorname{Diffeo}_{\text {bdy } E}^{+}(I)$ for maps that belong to $\operatorname{Diffeo}_{E}^{+}(I)$ and are fixed-point-free off $E$. (The details are in Subsection 2.13.)

The final theoretical step is the reduction of the conjugacy problem in $\operatorname{Diffeo}(I)$ to the conjugacy problem in $\operatorname{Diffeo}(I)^{+}$. (The details are in $\$ 9$ )

By a flow on an interval $I$, we mean a continuous homomorphism $t \mapsto \Phi^{t}$ from the additive topological group $(\mathbb{R},+)$ into $\operatorname{Diffeo}^{+}(I)$, endowed with its usual topology (the topology of simultaneous convergence of functions and their inverses, together with all their derivatives, uniformly on $I$ ).

We say that $f \in \operatorname{Diffeo}^{+}(I)$ is flowable if there exists a flow $\Phi^{t}$, with $f=\Phi^{1}$ (i.e., $f$ is the "time 1" map of the flow $\left.\left(\Phi^{t}\right)_{t \in \mathbb{R}}\right)$. 
There is a connection between our subject and the question of when an $f \in \operatorname{Diffeo}^{+}(I)$ is flowable. For this, see also [27]. We shall make some remarks about flowability as we go along (cf. Subsection 2.11).

Along the way, we present some conjectures and problems that, if true or solved, as the case may be, would improve our understanding of one-dimensional conjugacy.

\section{§2. OVERVIEW AND STATEMENT OF MAIN RESUltS}

2.1. Open intervals. A fixed-point-free diffeomorphism of an open interval $I$ must preserve orientation. There is just one conjugacy class of fixed-point-free diffeomorphisms in $\operatorname{Diffeo}(I)$, which splits into just two conjugacy classes with respect to $\operatorname{Diffeo}^{+}(I)$ :

Proposition 2.1 (Sternberg [28]). Suppose $I$ is an open interval and $f$ and $g$ are fixedpoint-free elements of $\operatorname{Diffeo}(I)$. Then $f$ and $g$ are conjugate in $\operatorname{Diffeo}^{+}(I)$ if and only if their graphs lie on the same side of the diagonal.

This is proved in $\$ 3$ below.

2.2. The interval $[0,+\infty)$. Note that $\operatorname{Diffeo}(I)=\operatorname{Diffeo}^{+}(I)$ whenever $I$ is a half-open interval, because all the elements of $\operatorname{Diffeo}(I)$ have to fix the endpoint that belongs to the interval.

Consider $f, g \in \operatorname{Diffeo}([0, \infty))$, fixed-point-free on $(0, \infty)$. Under what circumstances does there exist an $h \in \operatorname{Diffeo}([0, \infty))$ with $f=g^{h}$ ?

The set of all $f \in \operatorname{Diffeo}([0, \infty))$ that fix only 0 is the disjoint union of the two subsets

$$
\begin{aligned}
& S_{+}=\{f: f(x)>x \text { on }(0, \infty)\}, \\
& S_{-}=\{f: f(x)<x \text { on }(0, \infty)\},
\end{aligned}
$$

each of which is a subsemigroup of $\operatorname{Diffeo}([0, \infty))$. Each of these semigroups is preserved by conjugacy, i.e., is a union of conjugacy classes. Thus, for $f$ to be conjugated to $g$ it is necessary that they belong to the same semigroup, $S_{+}$or $S_{-}$. We call this the "sign condition".

Remark. In later sections, where the context changes, the meaning of the "sign condition" will change as well. So the above defines the sign condition just for the case of $\operatorname{Diffeo}([0,+\infty))$.

Note that $f \in S_{+}$is equivalent to $f^{\circ-1} \in S_{-}$, so that to characterize conjugacy it suffices to consider $f \in S_{-}$.

We review some special cases and then look at the general case.

2.3. $[0,+\infty)$ : Hyperbolic case. The result for the case $f^{\prime}(0) \neq 1$ is known as Sternberg's Linearization Theorem. It was essentially proved in 28. It may be regarded as the smooth equivalent of Schroeder's theorem [5, Chapter II] about complex analytic germs in one variable.

Theorem 2.2. Let $f, g \in S_{-}$and $f^{\prime}(0) \neq 1$. Then the following are equivalent:

(1) $f^{\prime}(0)=g^{\prime}(0)$

(2) there exists $h \in \operatorname{Diffeo}^{+}([0, \infty))$ with $f=g^{h}$;

(3) for each $\lambda>0$ the sequence $h_{n}=g^{\circ-n}\left(\lambda f^{\circ n}\right)$ converges (pointwise) to a diffeomorphism $h$ on $[0, \infty)$;

(4) the sequence $h_{n}=g^{\circ-n} \circ f^{\circ n}$ converges to a diffeomorphism $h$ on $[0, \infty)$;

(5) there exists $\lambda>0$ such that the sequence $h_{n}=g^{\circ-n}\left(\lambda f^{\circ n}\right)$ converges to a diffeomorphism $h$ on $[0, \infty)$.

The details are in Subsection 4.1. 
Corollary 2.3. If $f \in S$ and $f^{\prime}(0) \neq 1$, then the centralizer $C_{f}$ of $f$ in $\operatorname{Diffeo}([0,+\infty))$ is a one-parameter group and $f$ is flowable.

Proof. In fact, $C_{f}$ is the image under an inner automorphism of Diffeo $([0,+\infty))^{+}$of the centralizer of $x \mapsto f^{\prime}(0) x$, and this consists precisely of the maps $x \mapsto \mu x$ with $\mu>0$.

Also, the map $x \mapsto f^{\prime}(0)$ is the time 1 map of the flow $t \mapsto f_{t}$, where

$$
f_{t}(x)=e^{t \ln f^{\prime}(0)} x .
$$

2.4. $[0,+\infty)$ : Taylor series. Since all the elements fix 0 , we see that if $f=g^{h}$ in $\operatorname{Diffeo}^{+}([0,+\infty))$,

$$
T_{0} f=\left(T_{0} h\right)^{\circ-1} \circ\left(T_{0} g\right) \circ\left(T_{0} h\right) .
$$

Thus $T_{0} f$ and $T_{0} g$ are conjugate in the group of formally-invertible series. We call this Condition ( $\mathrm{T}$ ).

In case $f^{\prime}(0) \neq 1$, Condition (T) just says that $f^{\prime}(0)=g^{\prime}(0)$. In the nonhyperbolic case, it imposes conditions on some higher derivatives.

For the nonflat case, Takens [30, Theorem 2] proved the following theorem 1 ]

Theorem 2.4 (Takens). Suppose that $f, g \in S_{-}$and that $f-x$ is not flat at 0 . Then the following are equivalent:

(1) Condition (T) holds;

(2) there exists $h \in \operatorname{Diffeo}([0,+\infty))$ such that $f=g^{h}$.

Note that this generalizes the equivalence of (1) and (2) in Theorem 2.2, since the multiplier determines the conjugacy class of the series when it is not 1 .

See Subsection 4.2 for the details.

2.5. $[0,+\infty)$ : The case of $f-x$ flat at 0 . If $f-x$ is flat at 0 , Condition (T) just says that $g-x$ is also flat at 0 . This is not enough.

Example 2.1. Let $f(x)=x-e^{-\frac{1}{x}}$ and $g(x)=x-e^{-\frac{1}{x^{2}}}$. The functions $f$ and $g$ are not conjugate in Diffeo $([0,+\infty))$.

Proof. Suppose $h \in \operatorname{Diffeo}([0,+\infty))$, with Taylor series $T_{0} h=a X+b X^{2}+\cdots$, is a conjugation. Then it maps the interval $\left[\frac{x}{2}, x\right]$ to the interval $\left[\frac{a x}{2}+o(x), a x+o(x)\right]$. For small positive $x$, the first interval has no more than $x \exp (2 / x)$ iterates of $x$ under $f$, whereas the second has at least $\left.(x / 2) \exp \left(1 / 4 a^{2} x^{2}\right)\right)$ iterations of $h(x)$ under $g$, a much greater number. But the conjugacy condition requires that the two intervals contain equal numbers of iterates of $x$ and $h(x)$, respectively.

So we need another idea, in order to deal with two general elements $f, g \in S_{-}$. If you think about it, the main difficulty of the conjugacy problem of the present section involves the functions with $f-x$ flat at 0 . When endowed with the relative topology from the usual Fréchet-space topology on $C^{\infty}\left([0,+\infty)\right.$, the group $\operatorname{Diffeo}^{+}([0,+\infty))$ is separable and metrisable, so has the cardinality of the continuum, and hence (since Sternberg gives us a continuum of conjugacy classes) the family of conjugacy classes has the same cardinality. From this point of view, Sternberg's family is a substantial family of conjugacy classes.

However, cardinality is a very crude way to measure size. Another way is to use dimension. The map $D: f \mapsto f^{\prime}$ is a continuous bijection between Diffeo $([0,+\infty))$ and

\footnotetext{
${ }^{1}$ There is folklore that says that Mather independently found this result, but we checked with Mather, who said he definitely did not.
} 
the cone of all smooth positive functions $h$ on $[0,+\infty)$ that have

$$
\int_{0}^{\infty} h(x) d x=+\infty \text {. }
$$

This gives a way to embed our group as a convex subset in a Fréchet space and talk about the linear dimension and codimension of subvarieties. Sternberg's family is the complement of a codimension 1 variety, and so is a large part of the group. But consider the conjugacy classes. Conjugacy does not respect the convex structure of the cone (i.e., it does not commute with convex combinations), so we cannot induce a manifold structure on the conjugacy classes. What we can do is to measure the size of a family $\mathcal{F}$ of conjugacy classes in terms of the minimal dimension of $R$, where $R$ ranges over varieties that have at least one representative of each element of $\mathcal{F}$. Let's call this cardinal the conjugacy dimension of the family. From this point of view, Sternberg's family has conjugacy dimension 1.

If we take $G_{0}=$ Diffeo $^{+}([0,+\infty))$ and denote by $G_{n}$ the subgroup consisting of those $f \in G_{0}$ such that $f-x=o\left(x^{n}\right)$ at 0 , then we have a countable nested chain of closed normal subgroups

$$
\cdots \subset G_{n} \subset G_{n-1} \subset \cdots \subset G_{2} \subset G_{1} \subset G_{0}
$$

and each difference set $G_{n} \sim G_{n-1}$ has a naturally parametrized one-parameter family of conjugacy classes, so has conjugacy dimension one. Moreover, each difference is an open dense subset of the next group $G_{n-1}$, so it looks as though we have a nice stratification of the conjugacy classes, with just a trivial collection left at the core. But the fun really starts when we move inside the intersection $G_{\infty}$ of the chain. For instance, to each element $\phi \in G_{\infty} \cap S_{+}$we may associate a normal subgroup

$$
G_{\phi}=\left\{f \in G_{0}: f(x)-x=O(\phi(x))\right\} .
$$

One sees that the intersection of each countable family of groups $G_{\phi}$ is nontrivial, so by transfinite induction one can construct uncountable chains of $G_{\phi}$ 's. From the purely algebraic point of view, this is no different from what one can do inside the Sternberg family, because one can construct uncountable chains of normal subgroups by restricting the multiplier to subfields of the reals. But from the analytic point of view the $G_{\phi}$ are quite different groups, because their images under $D$ are cones and invariant under multiplication by positive reals. This makes it clear that there is no hope of tackling the conjugacy problem by reducing to explicit normal forms, since the set-theoretic difference of two normal subgroups is a union of conjugacy classes.

Neither is it possible to reduce it to the temptingly straightforward task of comparing vector fields whose exponentials are the given functions, for the simple reason that the exponential map is not surjective [27]. It is easy to check if two flows are related by a smooth change of variables, but not all diffeomorphisms are flowable.

The only way to come at it is to take two functions and compare them directly with one another, rather than with some collection of templates.

We find such a procedure by using a suitable infinite product and differential equation.

Arising from this discussion, we state a conjecture.

Conjecture. The conjugacy dimension of the diffeomorphism group of $[0,+\infty)$ is uncountable.

2.6. $[0,+\infty)$ : The product. Let us begin again, with general $f, g \in S_{-}$. For $x>0$ and $\xi>0$, let

$$
H_{1}(x, \xi)=H_{1}(f, g ; x, \xi)=\prod_{n=0}^{\infty} \frac{f^{\prime}\left(f^{\circ n}(x)\right)}{g^{\prime}\left(g^{\circ n}(\xi)\right)} .
$$


We say that $f$ and $g$ satisfy Condition (P) if there exist $x>0$ and $\xi>0$ such that the product $H_{1}(x, \xi)$ converges (to a nonzero limit).

The product $H_{1}(x, \xi)$ appears already in Sternberg's paper [28], in the special case $g(x)=\lambda x$, and in Kopell's paper [10] in the case $f=g$. We have not seen it used in the literature for general $f$ and $g$.

We shall show (Corollary 5.4) that if Condition (P) holds, then $H_{1}(x, \xi)$ exists for all $x>0$ and $\xi>0$, and (Lemma 6.1) is infinitely differentiable and positive. We may then consider the three-parameter initial-value problem

$$
D_{1}(a, \alpha, \lambda):\left\{\begin{array}{l}
\frac{d \phi}{d x}=H_{1}(x, \phi(x)) \lambda, \quad \forall x>0, \\
\phi(a)=\alpha
\end{array}\right.
$$

depending on $\lambda>0, a>0$ and $\alpha>0$. We shall show that for each given $a>0$ and $\alpha>0$, there exists (Lemma 6.11) exactly one $\lambda>0$ for which the (unique) solution $\phi$ to problem $D_{1}(a, \alpha, \lambda)$ has $f(a)=g^{\phi}(a)$, and (Lemma 6.9) that this $\phi$ conjugates $f$ to $g$ in Diffeo $\left((0,+\infty)\right.$ ), and (Lemma 6.10) extends in $C^{1}\left([0,+\infty)\right.$ ), with $\phi^{\prime}(0)=\lambda$. We denote this unique $\lambda$ by $\Lambda_{+}(a, \alpha)$, and the unique $\phi$ by $\Phi_{+}(a, \alpha)$.

Thus, subject to Condition (P), there is a 1-parameter family of $C^{1}$ conjugations from $f$ to $g$ on $[0,+\infty) 2$ This immediately gives us a result about $C^{\infty}$ conjugacy on $[0,+\infty)$.

Theorem 2.5 (Main Theorem). Suppose $f, g \in S_{-}$. The elements $f$ and $g$ are conjugate in Diffeo $([0,+\infty)$ if and only if Condition $(\mathrm{P})$ holds and there exists some $a>0$ and $\alpha>0$ for which $\Phi_{+}(a, \alpha)$ is $C^{\infty}$ at 0 .

The value of this result is that it narrows the search for a conjugating map $\phi$ to the 1-parameter family of solutions of an explicit ordinary differential equation.

We repeat (for emphasis) the fact already noted that when $f-x$ is not flat at 0 , then Condition (T) implies that $f$ is conjugate to $g$. Thus, since Condition (T) is easier to check than Condition $(\mathrm{P})$; the theorem is only interesting when $f-x$ is flat at 0 .

2.7. General half-open intervals. All the above results about $[0,+\infty)$ carry over to diffeomorphisms of an arbitrary half-open interval that fix only the endpoint that belongs to the interval. Each such interval is diffeomorphic to $[0,+\infty)$.

For a general half-open interval $I=[d, c)$ or $I=(c, d]$, we take $J=\operatorname{int}(I)$ and define $S_{-}$as the semigroup of diffeomorphisms $f \in \operatorname{Diffeo}(I)$ which iterate all points of $J$ towards the endpoint $d$, and $S_{+}$as the semigroup of those that iterate all points of $J$ towards $c$. In order to adapt the above results about $f, g \in S_{-}$to the interval $J \cup\{d\}$, one should replace $(0,+\infty)$ by $J$, and 0 by $d$. Then, for $f, g \in S_{-}$, the product Condition (P) takes precisely the same form (1), and the differential equation also, except that its domain is the interior $J$. The theorem yields, by conjugating $I$ to $[0,+\infty)$, a precisely similar result for $f, g \in S_{-}$on $I$.

For future reference, we formulate the condition (for two $f, g \in S_{-}$satisfying Condition $(\mathrm{P}))$.

Condition (E). There exist $a, \alpha \in J$, for which the $C^{1}$ extension of the solution $\phi=$ $\Phi_{+}(a, \alpha ; \bullet)$ from $J$ to the point $d$ is actually $C^{\infty}$.

It is equivalent to replace "there exist $a, \alpha$ " by "for each $a$ there exists $\alpha$ ".

In these terms, we may state the following.

Corollary 2.6. Let $I$ be half-open. Two elements $f, g \in S_{-}$are conjugate in $\operatorname{Diffeo}(I)$ if and only if they satisfy Conditions $(\mathrm{P})$ and $(\mathrm{E})$.

\footnotetext{
${ }^{2}\left\{\Phi_{+}(a, \alpha): a>0, \alpha>0\right\}$ is a 1-parameter family, because $\Phi_{+}(a, \alpha)=\Phi_{+}\left(b, \Phi_{+}(a, \alpha)(b)\right)$ for each $b>0$.
} 
Proof. This is immediate from Theorem 2.5.

For $f, g \in S_{+}$, one applies this result to $f^{\circ-1}$ and $g^{\circ-1}$, which lie in $S_{-}$. Unwinding the definitions, we see that Condition (P) for elements of $S_{+}$involves the infinite product

$$
H_{2}(x, \xi)=\prod_{n=1}^{\infty} \frac{g^{\prime}\left(g^{\circ-n}(\xi)\right)}{f^{\prime}\left(f^{\circ-n}(x)\right)}
$$

(for $x, \xi \in J$ ) and the differential equation takes the form:

$$
D_{2}(a, \alpha, \mu):\left\{\begin{array}{l}
\frac{d \phi}{d x}=H_{2}(x, \phi(x)) \mu, \text { on } J, \\
\phi(a)=\alpha,
\end{array}\right.
$$

for $a, \alpha \in J$ and $\mu>0$.

Assuming Condition (P), one has, for each for $a, \alpha \in J$, the existence of a unique $\mu>0$ (denoted $\Lambda_{-}(a, \alpha)$ ) for which the unique solution $\phi=\Phi_{-}(a, \alpha)$ has a $C^{1}$ extension to $d$, with $\phi^{\prime}(d)=\mu$. The version of Condition (E) for elements of $S_{+}$then says:

There exists $a, \alpha \in J$, for which the $C^{1}$ extension of the solution $\phi=\Phi_{-}(a, \alpha ; \bullet)$ from $J$ to the point $d$ is actually $C^{\infty}$.

With this terminology, the previous corollary yields:

Corollary 2.7. Let $I$ be half-open. Two elements $f, g \in S_{+}$are conjugate in $\operatorname{Diffeo}(I)$ if and only if they satisfy the $S_{+}$versions of Conditions $(\mathrm{P})$ and $(\mathrm{E})$.

2.8. Compact intervals. Now we consider the question of conjugacy for orientationpreserving diffeomorphisms of a compact interval $I$, which are fixed-point-free on the interior $J$.

Let $f$ and $g$ be two such diffeomorphisms.

The first necessary condition is the same as before:

The sign condition. $\operatorname{sign}(f(x)-x)=\operatorname{sign}(g(x)-x), \forall x \in J$.

This means that $f$ and $g$ are topologically conjugate, and have similar dynamics on $I$. Forward iteration from any point of $J$ converges monotonically to one end of $I$, and backward iteration leads to the other end. So the map $f$ induces a direction on $J-$ upward if $f(x)>x$ on $J$, downward if $f(x)<x$ on $J$. We label the ends of $J$ accordingly:

$$
\begin{aligned}
& d=d(J)=\lim _{n \rightarrow \infty} f^{\circ n}, \\
& c=c(J)=\lim _{n \rightarrow \infty} f^{\circ-n} .
\end{aligned}
$$

We call $c$ the "initial endpoint" of $J$, and $d$ its "final endpoint". We call the direction towards $d$ the "forward direction" on $J$, and the other the "backward direction".

For a compact interval $I=[c, d]$, with nonempty interior $J$, we define $S_{-}(I)$ as the semigroup of homeomorphisms that iterate each element of $J$ towards $d$.

In order that two given $f, g \in S_{-}$be conjugate in $\operatorname{Diffeo}([c, d])$, it is necessary that they be conjugate in $\operatorname{Diffeo}([c, d))$ and in Diffeo $((c, d]))$. Thus Corollaries 2.6 and 2.7 apply and tell us that the two-sided product

$$
H(x, \xi)=H(f, g, x, \xi)=H_{1}(x, \xi) / H_{2}(x, \xi)=\prod_{n=-\infty}^{\infty} \frac{f^{\prime}\left(f^{\circ n}(x)\right)}{g^{\prime}\left(g^{\circ n}(\xi)\right)}
$$

must converge for some (or equivalently all) $x, \xi \in J$. This is the appropriate version of Condition ( $\mathrm{P})$, for compact intervals.

Assuming Condition (P), we may form two initial-value problems, corresponding to equations (2) and (41). Given $a \in J$ and $\alpha \in J$, there are unique $\lambda$ and $\mu$, respectively, 
A. G. O'FARRELL AND M. ROGINSKAYA

such that the solutions $\Phi_{+}(a, \alpha)$ and $\Phi_{-}(a, \alpha)$, respectively, to these equations conjugate $f$ to $g$ on $J$ and have $C^{1}$ extensions to $(c, d]$ and $[c, d)$, respectively. We may then formulate a solution to the conjugacy problem, as follows:

Theorem 2.8. Let $I$ be a compact interval and let $f, g \in \operatorname{Diffeo}(I)$, both fixed-point-free on $J$, both in $S_{-}$. Then the following conditions are equivalent:

(1) $f$ is conjugate to $g$ in $\operatorname{Diffeo}(I)$;

(2) the product $H(x, \xi)$ converges for some (and hence for all) $x>0$ and $\xi>0$, and there exists some $a>0$ and $\alpha>0$ such that the solution $\Phi_{+}(a, \alpha)$ extends $C^{\infty}$ to both ends of $I$;

(3) there exist $a>0$ and $\alpha>0$ such that $H(a, \alpha)$ converges, and $\Phi_{+}(a, \alpha)=\Phi_{-}(a, \alpha)$ extends in $\operatorname{Diffeo}(I)$.

The details are in $\$ 7$

2.9. Compact $I$ : Functional moduli. For some problems of classification, a solution is available in terms of a finite-dimensional space of invariants, or "moduli". But if the class structure is very rich, this may not be possible, and only infinite-dimensional spaces of moduli are natural. This is the genesis of the idea of functional moduli (cf. 31).

In special cases, the conjugacy problem on a compact interval can be reduced to Condition ( $\mathrm{T}$ ) at both ends, plus the identity of a suitable modulus (a conjugacy invariant that is a diffeomorphism on some interval). See Robbin [25], Afraimovitch, Liu and Young [1], and Young [34]. All these results are subsumed in an unpublished lemma of Mather [15, subsequently and independently found by Young, which covers the case in which the germs of $f$ at both ends of the interval are the exponentials of smooth vector fields, and for which the modulus is a double coset $R k R$ of the rotation group $R=\mathrm{SO}(2, \mathbb{R})$ in the group Diffeo ${ }^{+}\left(\mathbb{S}^{1}\right)$ of circle diffeomorphisms, and the conjugacy class of $f$ is determined by the smooth conjugacy classes of the two vector field germs and the modulus.

See Subsection 7.4 for more details on the moduli.

2.10. Compact $I$ : Shape. Obviously, it is rather unlikely that two given maps $f$ and $g$ will be smoothly conjugate on $I$, even assuming they satisfy the sign condition and Condition (P).

The conditions of Theorem 2.8 are necessary and sufficient, but are tedious to check.

It is worth noting a necessary condition (the "shape" condition) that is easier to check in the compact case. This will often suffice to show that two maps are not conjugate.

First we define

$$
F_{a}(x)=H(f, f ; x, a)=\prod_{n=-\infty}^{\infty} \frac{f^{\prime}\left(f^{\circ n}(x)\right)}{f^{\prime}\left(f^{\circ n}(a)\right)}
$$

and

$$
G_{\alpha}(\xi)=H(g, g ; \xi, \alpha)=\prod_{n=-\infty}^{\infty} \frac{g^{\prime}\left(g^{\circ n}(\xi)\right)}{g^{\prime}\left(g^{\circ n}(\alpha)\right)}
$$

whenever $x, \xi, a, \alpha \in J$. Note that

$$
H(x, \xi) \cdot G_{\alpha}(\xi)=F_{a}(x) \cdot H(a, \alpha),
$$

whenever all the terms make sense.

Proposition 2.9. Suppose $f, g, h \in \operatorname{Diffeo}(I), f$ is fixed-point-free on $J$, and $f=g^{h}$. Then $H(x, h(x))$ is constant on $J$. Thus, given any $a, \alpha \in J$, there is some $\kappa>0$ such that

$$
F_{a}(x)=\kappa G_{\alpha}(h(x)), \forall x \in J .
$$


Proof. Suppose $I$ is compact, $f \in S_{-}$, and $f=g^{h}$ in Diffeo $(I)$. Applying the results about half-open intervals to both $[c, d)$ and $(c, d]$, we see that the product $H_{1}(x, h(x))$ converges to $h^{\prime}(x) / h^{\prime}(d)$ for each $x \in J$, and $H_{2}(x, h(x))$ converges to $h^{\prime}(x) / h^{\prime}(c)$ for each $x \in J$. Thus the two-sided product

$$
H(x, h(x))=H_{1} / H_{2}=\prod_{n=-\infty}^{\infty} \frac{f^{\prime}\left(f^{\circ n}(x)\right)}{g^{\prime}\left(g^{\circ n}(h(x))\right)}
$$

is independent of $x \in J$, and equals the ratio $h^{\prime}(c) / h^{\prime}(d)$ of the derivatives of the conjugating map at the ends.

This immediately tells us that $H(x, h(x))$ is constant. The rest then follows from equation (6).

This means that the graphs of each $F_{a}$ and of each $G_{\alpha}$ have the same "shape". If they are not monotone, then the relative diffeomorphism class of the critical set and the pattern of maxima and minima must be the same for both functions. The pattern for $F_{a}$ is determined by the pattern on the segment $I_{a}=[a, f(a)]$, because it repeats itself on successive images of $I_{a}$ under $f$. Similarly, the pattern for $G_{\alpha}$ is determined by the pattern on $[\alpha, g(\alpha)]$. Apart from this quasiperiodic feature, the patterns may be pretty complicated.

Note that if the condition of the proposition fails, then this can be determined by a computation.

2.11. Compact $I$ : Flowability. We note applications to existence of a smooth flow on a compact interval $I=[c, d]$, for which $f$ is the time-1 step.

Applying Theorem 2.8 to the case $g=f$, we see that the centralizer $C_{f}$ is the intersection of two at-most-one-parameter groups, containing the (nontrivial, discrete) group of all iterates of $f$. (It may well be that only the compositional powers of $f$ belong to $C_{f}$.)

We deduce a method for deciding whether or not $f$ is the time 1 map of a flow.

Proposition 2.10. A diffeomorphism $f \in \mathrm{Diffeo}^{+}(I)$ is flowable if and only if the centralizers of $f$ in $\operatorname{Diffeo}(\{c\} \cup J)$ and $\operatorname{Diffeo}(J \cup\{d\})$ are both connected, and coincide (when restricted to $J$ ).

Applying the shape result, Proposition 2.11, we identify a special case in which a necessary condition for flowability may be checked by plotting a graph.

Proposition 2.11. Suppose that $f \in \operatorname{Diffeo}(I)$ is fixed-point-free on $J$ and $f$ is flowable. Then for each $a \in J, F_{a}$ is either strictly monotone on $J$ or constant on $J$.

Proof. Suppose that $F_{a}$ is neither strictly monotone on $J$ nor constant on $J$. Each conjugacy of $f$ to itself must permute the maximal open intervals of strict monotonicity of $F_{a}$. Since $F_{a}$ is smooth and not strictly monotone or constant, there exist at least two such intervals, and since the pattern repeats, there are in fact infinitely many. But the number is countable, since they are pairwise disjoint open sets, and conjugacy must permute the countable set of endpoints of these intervals of monotonicity and is determined uniquely by the image of one endpoint. Hence the centralizer of $f$ is a countable group, so $f$ cannot be flowable.

We can do better when the graph of $f$ is tangent to the diagonal at the ends of $I$.

Corollary 2.12. Suppose $f \in \operatorname{Diffeo}(I)$ is fixed-point-free on $J$ and is flowable. Then the following are equivalent:

(1) $f^{\prime}(c)=f^{\prime}(d)$; 
(2) $f^{\prime}(c)=f^{\prime}(d)=1$

(3) $F_{a}$ is constant on $J$, for each (or any one) $a \in J$.

Proof. The implication (1) $\Longrightarrow$ (2) follows from the fact that 1 is always trapped between $f^{\prime}(c)$ and $f^{\prime}(d)$.

Next, note that we have the formula

$$
F_{a}(f(x))=F_{a}(x) \frac{f^{\prime}(d)}{f^{\prime}(c)},
$$

whenever $a, x \in J$.

Suppose (2) holds. Fix $a \in J$. The formula (7) implies that $F_{a}(f(x))=F_{a}(x)$ for all $x \in J$. Since $f$ is flowable, Proposition 2.11 tells us that $F_{a}$ is constant on each interval $[f(x), x]$. But for any fixed $x_{0}=x$, the iterates $x_{n}=f^{\circ n}(x)$ converge monotonically to one end of $J$ as $n \uparrow+\infty$, and monotonically to the other end as $n \downarrow-\infty$; hence the intervals $\left[x_{n+1}, x_{n}\right]$ pave $J$, and, since $F_{a}$ is constant on each, it is constant on the whole interval $J$. Thus $(2) \Longrightarrow(3)$.

Finally, suppose (3) holds. Then equation (17), applied to any $x \in J$, yields $f^{\prime}(d)=$ $f^{\prime}(c)$, since $F_{a}(x)$ never vanishes.

We note that these results depend only on the assumption that $f \in C^{2}(I)$.

2.12. Conjugacy in $\operatorname{Diffeo}^{+}(I)$. Now we move on to the general orientation-preserving case on an arbitrary interval $I \subset \mathbb{R}$.

Each interval is diffeomorphic to one of the closed intervals $\mathbb{R},[0,+\infty)$ or $[-1,1]$, so there is no loss in generality in supposing that $I$ is a closed interval. (If $I$ is not closed, fix some diffeomorphism $h$ of $I$ onto a closed interval. Then $f$ and $g$ are conjugate in Diffeo $^{+}(I)$ if and only if ${ }^{h} f$ and ${ }^{h} g$ are conjugate in Diffeo ${ }^{+}(h(I))$.)

We can reduce the problem to the conjugacy problem in $\operatorname{Diffeo}_{B}^{+}=\operatorname{Diffeo}_{B}^{+}(I)$, with $B=$ bdy $E$ and $E \subset I$ closed.

Proposition 2.13. Suppose $I$ is a closed interval. Let $f, g \in$ Diffeo $^{+}$. Then there exists $h \in$ Diffeo $^{+}$such that $f=g^{h}$ if and only if there exists $h_{1} \in$ Diffeo $^{+}$such that the following three conditions hold:

(1) $h_{1}(\operatorname{fix}(f))=\operatorname{fix}(g)$;

(2) letting $f_{1}=f^{h_{1}^{\circ-1}}$ and $E=\operatorname{fix}(g)$, we have

$$
\operatorname{sign}\left(f_{1}(x)-x\right)=\operatorname{sign}(g(x)-x), \forall x \in I \sim E ;
$$

(3) there exists $h_{2} \in$ Diffeo $_{\text {bdy } E}^{+}$such that $f_{1}=g^{h_{2}}$.

Proof. "Only if": Suppose there exists $h \in$ Diffeo $^{+}$such that $f=g^{h}$.

Taking $h_{1}=h$, we have condition (1). Also, in that case $f_{1}=g$, so condition (2) holds. Taking $h_{2}=I d$, we have condition (3).

"if": Suppose there exist $h_{1}$ and $h_{2}$ satisfying conditions (1), (2) and (3). Then $h=h_{2} \circ h_{1}$ has $f=g^{h}$.

As already remarked, the existence of an $h$ with condition (1) is not amenable to algorithmic checking, so we shall just treat it as a primitive condition.

Given the existence of such an $h$, there may exist many. Condition (2) cuts down the collection of eligible $h$. One then has to check condition (3) for each eligible $h$. In this sense, we have reduced the conjugacy problem in Diffeo ${ }^{+}$to the problem of characterizing conjugacy in Diffeo ${ }_{\text {bdy } E}^{+}$, for two elements of Diffeo ${ }_{E}^{+}$(see Example 2.2). It is worth remarking that the condition that $T_{a} f$ and $T_{h(a)} g$ be conjugate Taylor series reduces the collection of eligible $h$ considerably. See further remarks in Subsection 10.1. 
Obviously, the reduction achieved here is not deep. However it is useful. If we replace bdy $E$ by $E$ in condition (3), then the restated proposition remains true, but is less useful. To explain this point, consider this example:

Example 2.2. Take $I=\mathbb{R}$ and set

$$
g(x)= \begin{cases}x, & x \leq 0 \\ x+e^{-1 / x}, & x>0,\end{cases}
$$

and $f(x)=\frac{1}{2}(1+g(2 x-1))$. Then $x \mapsto 2 x-1$ conjugates $f$ to $g$ in Diffeo ${ }^{+}$.

If we are handed two functions $f$ and $g$ in Diffeo ${ }^{+}$, and asked to determine whether or not they are conjugate, then we would begin by comparing the pairs $(\mathbb{R}, \operatorname{bdy}(\operatorname{fix}(f)))$ and $(\mathbb{R}, \operatorname{bdy}(\operatorname{fix}(g)))$ to see whether they lie in the same diffeomorphism class. In the present example, the pairs are $\left(\mathbb{R},\left\{\frac{1}{2}\right\}\right)$ and $(\mathbb{R},\{0\})$, and (of course) they do. The next reasonable step would be to take any $h_{1} \in$ Diffeo $^{+}$that maps $\frac{1}{2}$ to 0 , and replace $f$ by $f_{1}=f^{h_{1}^{\circ-1}}$, as in condition (2) of the proposition, and proceed to compare $f_{1}$ and $g$, which now have the same fixed point set, namely $E=(-\infty, 0]$. Let's say we chose (slightly perversely), $h_{1}(x)=4 x-2$. Then we would have $f_{1}(x)=g(x)=x$ for $x \leq 0$, and $f_{1}(x)=2 g(x / 2)$ for $x>0$. We would then proceed to check that $f_{1}$ and $g$ are conjugate, and it would be reasonable to seek a conjugacy on the lines of (3), that fixes 0 . We could then use the differential equation, as in Section 4 to "discover" one of the conjugacies that exist in Diffeo $([0,+\infty))$ (i.e., one of the elements of the coset of $C_{f}$ to which $x \mapsto 2 x$ belongs). Now each such conjugacy is $C^{\infty}$ down to 0 and has derivative 2 at 0 . Extending it in any way whatsoever to a diffeomorphism of $(-\infty, 0]$ gives a global conjugacy $h_{2}$ from $f_{1}$ to $g$, because both maps are the identity map on the negative axis and are conjugated by anything. Each $h_{2}$ found in this way fixes $\{0\}$ but, and this is the point, there is no conjugacy of $g$ to $f_{1}$ that fixes all points of $E$. In order to attack the problem in this way it is essential to retain the flexibility to move points inside fix $(g)$. Otherwise, this approach goes nowhere.

2.13. Conjugacy in $\operatorname{Diffeo}_{B}^{+}$. Throughout this subsection, $I$ will be a fixed closed interval (bounded or not), $E$ will be a fixed closed nonempty subset of $I$, containing all endpoints of $I$, and $B$ will be the boundary of $E$.

We consider $f, g \in \operatorname{Diffeo}^{+}(I)$. (Recall that in view of Proposition 2.1 we need not consider the special case $E=\varnothing$.)

As before, we suppress the explicit $(I)$ in $\operatorname{Diffeo}^{+}(I), \operatorname{Diffeo}_{E}^{+}(I), \operatorname{Diffeo}_{B}^{+}(I)$, etc. $I \sim E$ is a countable union of open intervals. The following is trivial.

Proposition 2.14. Let $f, g \in$ Diffeo $^{+}$, fixing precisely $E$. Then $f$ is conjugate to $g$ in Diffeo $_{B}^{+}$if and only if there is a global function $h \in$ Diffeo $^{+}$such that, for each connected component $J$ of $I \sim E$, the restriction to each $\operatorname{clos}(J)$ belongs to $\operatorname{Conj}(f, g ; J)$.

So, necessary conditions for the conjugacy are as follows.

(1) If $J$ is an unbounded component of $I \sim E$, then the restrictions of $f$ and $g$ to the closure of $J$ satisfy the conditions of Corollary 2.6 or Corollary 2.7, as appropriate (i.e., depending on whether or not $f$ iterates points towards or away from the (finite) end of $J$ ).

(2) If $J$ is a bounded component of $I \sim E$, then the restrictions of $f$ and $g$ to the closure of $J$ satisfy the conditions of Theorem 2.8 .

(3) If an endpoint $p=c(J)$ or $d(J)$ is not isolated in $B$, then some conjugating $h \in \operatorname{Diffeo}_{B}^{+}$must have $h-x$ flat at $p$.

Condition (3) actually implies that all elements of $\operatorname{Conj}(f, g ; J)$ must be flat at $p$. 
Lemma 2.15. Suppose $f$ is conjugate to $g$ in Diffeo $_{B}^{+}$. Then whenever an end $c(J)$ or $d(J)$ of a component $J$ is an accumulation point of $B$, it follows that each $\phi \in$ Diffeo $^{+}(\operatorname{clos}(J))$ that conjugates $f$ to $g$ on $I$ has $\phi(x)-x$ flat there.

The proof is in $₫ 8$.

So we formulate this version of condition (3).

Condition (F). If $J$ is a connected component of $I \sim E$ and an endpoint $p=c(J)$ or $d(J)$ is not isolated in $B$, then each conjugating $h \in \operatorname{Diffeo}_{B}^{+}$must have $h-x$ flat at $p$.

But the conditions (1)-(3) will not always guarantee the existence of a global conjugation, even when $E$ is finite, or, more generally, discrete. The family $\operatorname{Conj}(J)=$ $\operatorname{Conj}(f, g ; J)$ is mapped by $h \mapsto T_{p} h$ to a set $M(p, J)=M(f, g ; p, J)$ of Taylor series, whenever $p$ is a finite end of $J$. This set $M(p, J)$ is a coset of a subgroup of the invertible Taylor series, and is, generically, discrete. Whenever two intervals $J$ and $J^{\prime}$ have a common endpoint $p$, we are snookered unless $M(p, J)$ and $M\left(p, J^{\prime}\right)$ intersect. This gives us a necessary condition.

Condition $\left(\mathbf{M}_{1}\right)$. If $p$ is an isolated point of $B$, and $J$ and $J^{\prime}$ are the components of $\mathbb{R} \sim E$ to the left and right of $p$, then $M(p, J) \cap M\left(p, J^{\prime}\right) \neq \varnothing$.

In case $p$ is a hyperbolic fixed point, Condition $\left(\mathrm{M}_{1}\right)$ is equivalent to the simpler condition that the multiplier cosets $\left\{h^{\prime}(p): h \in \operatorname{Conj}(f, g, J)\right\}$ intersect. (These are cosets of a subgroup of the multiplicative group $(0, \infty)$.) For suppose we take conjugating maps $h$ and $k$ on $\operatorname{clos} J$ and clos $J^{\prime}$, respectively, with the same multiplier at $p$. Then $T_{p} h$ conjugates $T_{p} f$ to $T_{p} g$, and so does $T_{p} k$, so $\left(T_{p} h\right) \circ\left(T_{p} k\right)^{\circ-1}$ commutes with $T_{p} f$ and has multiplier 1. Now a hyperbolic series is conjugate to its linear part, and the centralizer of a linear series is the set of all linear series, so an element of the centralizer of a hyperbolic series is determined uniquely by its multiplier. Hence $\left(T_{p} h\right) \circ\left(T_{p} k\right)^{\circ-1}$ equals $X$.

A similar argument shows that if $T_{p} f=X \bmod X^{p}$, but $T_{p} f \neq X \bmod X^{p+1}$, then Condition $\left(\mathrm{M}_{1}\right)$ simplifies to the condition that the quotients $\bmod X^{p+1}$ intersect:

$$
\left(M(p, J) \bmod X^{p+1}\right) \cap\left(M\left(p, J^{\prime}\right) \bmod X^{p+1}\right) \neq \varnothing .
$$

Example 2.3. Take $f$ and $g$, fixing only 0 , with $f-x$ and $g-x$ flat at 0 , such that $f$ is conjugated to $g$ on $J_{1}=[0,+\infty)$ by $x \mapsto 2 x$, and $f=g$ on $J_{2}=(-\infty, 0]$. Then $\operatorname{Conj}\left(f, g, J_{1}\right)$ is nonempty, but only has maps with derivative 2 at 0 , whereas $\operatorname{Conj}\left(f, g, J_{2}\right)$ has only maps with derivative 1 at 0 , so Condition $\left(\mathrm{M}_{1}\right)$ fails.

Assuming Condition $\left(\mathrm{M}_{1}\right)$, we have a further problem if there is a chain of successive isolated points in $E$. We then have a chain $J_{1}, \ldots, J_{k}$ of successive components of $I \sim E$. To find a conjugation, we must patch together elements of the $\operatorname{Conj}\left(f, g ; J_{i}\right)$ to make a single smooth conjugation on the closure of the union of the $J_{i}$. If we begin with one element of $\operatorname{Conj}\left(f, g ; J_{1}\right)$, and work along, trying to match its Taylor series at each common endpoint, then it becomes increasingly improbable that we will succeed. If there is any chain of intervals for which it cannot be done, then there is no global conjugation.

The key to further progress is to focus on $B^{\prime}$, the set of accumulation points of $B$. The connected components of $I \sim B^{\prime}$ include the connected components $J$ of the interior of $E$, and on these $J$ every diffeomorphism conjugates $f$ to $g$. This makes it reasonable to define $\operatorname{Conj}(f, g ; J)=\operatorname{Diffeo}^{+}(\operatorname{clos} J)$ for such a $J$.

We formulate a stronger version of Condition $\left(\mathrm{M}_{1}\right)$.

Condition $\left(\mathrm{M}_{2}\right)$. Given any connected component $L$ of $\mathbb{R} \sim B^{\prime}$, there exists a function $h \in \operatorname{Diffeo}^{+}(L)$ whose restriction to each component $J$ of $L \sim B$ belongs to $\operatorname{Conj}(f, g ; J)$. 
Each set $L \cap B$ is empty, or finite, or forms a sequence tending to one end of $L$, or a two-sided sequence accumulating at both ends of $L$.

If the condition fails, then it can be disproved by starting in any one $J$, and working left and right, cutting down the set of eligible conjugations until at some stage it is found that the set is empty.

However, it may be that all the functions that thread together conjugations on the various $J$ wiggle too much to extend smoothly to the accumulation points at the ends. So we formulate:

Condition (M). Given any connected component $L$ of $I \sim B^{\prime}$, there exists a function $h \in \operatorname{Diffeo}^{+}(\operatorname{clos} L)$ whose restriction to each component $J$ of $L \sim B$ belongs to $\operatorname{Conj}(f, g ; J)$.

Note that this implies conditions (1) and (2).

It may seem that we are heading into a jungle as complex as that involved in the order-equivalence problem and that higher derived sets are about to appear. But the situation is not so bad. We do not have to look at $B^{\prime \prime}$.

Theorem 2.16. Let I be a closed interval. Let $f, g \in \operatorname{Diffeo}^{+}(I)$ both fix precisely $E \subset I$. Then $f$ is conjugate to $g$ in $\operatorname{Diffeo}^{+}(I)$ (or, equivalently, in $\operatorname{Diffeo}_{B}^{+}(I)$ ) if and only if both Conditions (M) and (F) hold.

The proof is in 88 .

2.14. Reducing from $\operatorname{Diffeo}(I)$ to $\operatorname{Diffeo}^{+}(I)$. In 99 we discuss the reduction of the conjugacy problem in the full diffeomorphism group to the conjugacy problem in the subgroup of direction-preserving maps.

We close with some examples in $\$ 10$.

\section{$\S 3$. The FIXED-POINT-FREE CASE}

In this section our main purpose is to prove Proposition 2.1 Some of the elements of the proof will be useful later, for other purposes.

So suppose $f$ and $g$ are fixed-point-free elements of $\operatorname{Diffeo}^{+}(\mathbb{R})$, and both move all points up, or both move all points down. Then we have to show that $f$ and $g$ are conjugate in $\operatorname{Diffeo}^{+}(\mathbb{R})$.

The proof depends on a well-known result due to É. Borel (cf. [18, or [26, Chapter 19].

Theorem 3.1. For each $a \in \mathbb{R}$, each formal power series is the power series at a of some smooth function.

Corollary 3.2. Given a point $a \in \mathbb{R}$, any value $\lambda \in \mathbb{R}$, and a power series $P=a_{1} X+\cdots$ with $a_{1}>0$, there exists $f \in$ Diffeo $^{+}$with truncated Taylor series $T_{a} f=P$, and with $f(a)=\lambda$.

Proof. First, pick a smooth function $h_{1}$ with Taylor series at $a$ equal to the term-by-term derivative $P^{\prime}$ of $P$. Then $h_{1}$ will be positive near $a$, so by modifying it off a neighborhood of $a$ one may construct an everywhere-positive smooth function $h_{2}$ with the Taylor series $P^{\prime}$ at $a$. Now take

$$
f(x)=\lambda+\int_{a}^{x} h_{2}(t) d t, \quad \forall x \in \mathbb{R} .
$$

Corollary 3.3. Given real numbers $a<b$ and formal series

$$
P=a_{1} X+\cdots, \quad Q=b_{1} X+\cdots
$$


with $a_{1}>0, b_{1}>0$, there exists $f \in$ Diffeo with

$$
f(a)=a, \quad f(b)=b, \quad T_{a} f=P, \quad \text { and } \quad T_{b} f=Q .
$$

Proof. Applying the previous corollary twice, choose diffeomorphisms $r$ and $s$ such that

$$
\begin{array}{ll}
r(a)=a, & T_{a} r=P, \\
s(b)=b, & T_{b} s=Q .
\end{array}
$$

Since $r^{\prime}(a)>0$ and $s^{\prime}(b)>0$, we may choose $\eta>0$ such that $r$ maps $[a, a+2 \eta]$ diffeomorphically onto $[a, r(a+2 \eta)], s$ maps $[b-2 \eta, b]$ diffeomorphically onto $[s(b-2 \eta), b]$, and

$$
\max \{a+2 \eta, r(a+2 \eta)\}<\min \{b-2 \eta, s(b-2 \eta)\} .
$$

Choose a monotonically nonincreasing smooth function $t$ that is identically 1 on $(-\infty, a+\eta]$ and is identically 0 on $[a+2 \eta,+\infty)$.

Choose a monotonically nondecreasing smooth function $u$ that is identically 0 on $(-\infty, b-2 \eta]$ and is identically 1 on $[b-\eta,+\infty)$.

Choose another smooth function $v$ that is nonnegative, is not identically zero, but is zero off $[a+\eta, b-\eta]$.

For each $\lambda>0$, let

$$
h_{\lambda}(x)=t(x) \cdot r^{\prime}(x)+u(x) \cdot s^{\prime}(x)+\lambda \cdot v(x), \quad \forall x \in \mathbb{R} .
$$

Then $h_{\lambda}(x)>0$ for all $x \in \mathbb{R}, h_{\lambda}(x)=r^{\prime}(x)$ whenever $x<a+\eta, h_{\lambda}(x)=s^{\prime}(x)$ whenever $x>b-\eta$.

Define

$$
f_{\lambda}(x)=a+\int_{a}^{x} h_{\lambda}(z) d z, \quad \forall x \in \mathbb{R} .
$$

Then $f_{\lambda}$ is a diffeomorphism and fixes $a$. Also $f_{\lambda}$ has truncated Taylor series $P$ at $a$ and $Q$ at $b$. To finish, we just need to pick $\lambda>0$ so that $f_{\lambda}(b)=b$. This can be done, because it amounts to solving

$$
\lambda \int_{a}^{b} v(z) d z=b-a-\int_{a}^{a+2 \eta} t(z) r^{\prime}(z) d z-\int_{b-2 \eta}^{b} u(z) s^{\prime}(z) d z
$$

and the right-hand side is positive, since it exceeds

$$
b-a-\int_{a}^{a+2 \eta} r^{\prime}(z) d z-\int_{b-2 \eta}^{b} s^{\prime}(z) d z=s(b-2 \eta)-r(a+2 \eta)>0 .
$$

Now we can give the proof of Proposition 2.1.

Proof. Since $\mathbb{R}$ is diffeomorphic to each nonempty open interval $I \subset \mathbb{R}$, it suffices to prove Proposition 2.1 for the case $I=\mathbb{R}$.

By means of a preliminary conjugation with a linear map, we may assume that $g(0)=$ $f(0)$. Let $b=f(0)$.

Pick a smooth increasing map $\varphi$ of the interval $[0, b]$ onto itself such that

$$
\left(T_{b} \varphi\right) \circ\left(T_{0} f\right)=\left(T_{0} g\right) \circ\left(T_{0} \varphi\right) .
$$

(For instance, one could take $T_{0} \varphi \equiv X$ and let the above equation define $T_{b} \varphi$; the existence of a $\varphi$ matching these Taylor series follows from the last corollary.)

The conjugacy equation then forces a unique extension of $\varphi$ up to an element of the set $\operatorname{Diffeo}^{+}(\mathbb{R})$. 
We see that there are a great many conjugacies between two given conjugate diffeomorphisms. In particular, the centralizer of a fixed-point-free diffeomorphism is very large and is not Abelian. We shall see below that the presence of even a single fixed point produces a drastic reduction in the size of the centralizer. It becomes at most a one-parameter Abelian group.

\section{$\S 4 .[0,+\infty)$ : NECESSARY CONDITIONS}

\subsection{Proof of Theorem 2.2,}

Proof. Obviously, (2) implies (1).

(1) implies (3): Let $f^{\prime}(0)=g^{\prime}(0)=a$. Observe that, as $f \in S_{-}, 0<a<1$. By Sternberg there exist $\phi, \psi \in \operatorname{Diffeo}^{+}([0, \infty))$ such that $\phi^{-1} \circ f \circ \phi(x)=a x=\psi^{-1} \circ g \circ \psi(x)$. Now, if $\tau_{a}(x)=a x$, the functions of the sequence can be presented as $h_{n}=\psi \circ \tau_{a}^{-n} \circ$ $\psi^{-1} \circ \tau_{\lambda} \circ \phi \circ \tau_{a}^{n} \circ \phi^{-1}$. As both $\phi$ and $\psi$ are diffeomorphisms, $\phi(x)=\phi^{\prime}(0) x+O\left(x^{2}\right)$, and $\psi^{-1}(x)=\left(\psi^{-1}\right)^{\prime}(0) x+O\left(x^{2}\right)$. This means that $\phi \circ \tau_{a}^{n} \circ \phi^{-1}(x)=\phi^{\prime}(0) a^{n} \phi^{-1}(x)+$ $O\left(\left(a^{n} \phi^{-1}(x)\right)^{2}\right)$, and $\psi^{-1} \circ \tau_{\lambda} \circ \phi \circ \tau_{a}^{n} \circ \phi^{-1}=\left(\psi^{-1}\right)^{\prime}(0) \lambda \phi^{\prime}(0) a^{n} \phi^{-1}(x)+O\left(\left(a^{n} \phi^{-1}(x)\right)^{2}\right)$. Placing this expression in the formula for $h_{n}$ we get $h_{n}(x)=\psi\left(\left(\psi^{-1}\right)^{\prime}(0) \lambda \phi^{\prime}(0) \phi^{-1}(x)+\right.$ $\left.O\left(a^{n} \phi^{-1}(x)\right)\right)$. For a fixed $x$ we see that $h_{n}(x) \rightarrow \psi\left(\left(\psi^{-1}\right)^{\prime}(0) \lambda \phi^{\prime}(0) \phi^{-1}(x)\right)$, when $n \rightarrow \infty$, which is a diffeomorphism.

Obviously, (3) implies (4), and (4) implies (5).

(5) implies (2): We have $g \circ h(x)=g\left(\lim \left(h_{n}(x)\right)\right)=\lim g\left(h_{n}(x)\right)=\lim h_{n-1}(f(x))=$ $h \circ f(x)$, and $h \in \operatorname{Diffeo}^{+}([0, \infty))$.

The nice thing about this is that (3)-(5) give us a construction for conjugacy maps. Ahern and Rosay call the construction (4) "the basic trick".

4.2. Proof of Theorem 2.4. Any two elements of $S_{-}$that agree on a neighborhood of 0 are smoothly conjugate. This is easy to see: one just uses the conjugacy equation to extend the trivial conjugation given by the identity function near 0 to a smooth conjugacy on the whole of $[0,+\infty)$.

So, to prove Taken's conjugacy theorem we must show that if $f \in \operatorname{Diffeo}([0,+\infty))$ and the series $T_{0} f$ is conjugate to $X-X^{p+1}+\alpha X^{2 p+1}$, then $f$ is smoothly conjugate to a diffeomorphism that coincides with $g=x-x^{p+1}+\alpha x^{2 p+1}$ for small enough $x>0$.

However, the constructive method of the proof of Theorem 2.2 cannot be used directly in this case, in order to find a conjugacy.

Example 4.1. Consider

$$
f(x)=x+x^{2}, \quad \forall x \geq 0 .
$$

This function belongs to $S_{+}$and is conjugate to

$$
g(x)=x+2 x^{2}=\frac{1}{2} f(2 x),
$$

and hence the conjugating map $h: x \mapsto \frac{1}{2} x$ conjugates $f^{\circ-1} \in S_{-}$to $g^{\circ-1}$. But the conjugation functions in general cannot be recovered as in condition 3 of Theorem 2.2 . For instance, taking $\lambda=2$, we find that

$$
g^{\circ n}\left(2 f^{\circ-n}(x)\right) \rightarrow \infty
$$

and does not converge at all. One can see this by observing that

$$
g^{\circ n}\left(2 f^{\circ-n}(x)\right)=\frac{1}{2} f^{\circ n}\left(4 f^{\circ-n}(x)\right),
$$

due to the conjugation. On the other hand given a small number $x$, the first number $N$ for which $f^{\circ N}(x) \geq 4 x$ is at least $\frac{3 x}{(4 x)^{2}}=\frac{3}{16 x}$, and as the functions are monotone, we 
see that $f^{\circ n}\left(4 f^{\circ-n}(x)\right) \geq f^{\circ\left(N_{n}\right)}(x)$, where $N_{n}=\left\lfloor\frac{3}{16 f^{\circ-n}(x)}\right\rfloor \rightarrow \infty$ when $n \rightarrow \infty$. Thus $g^{\circ n}\left(2 f^{\circ-n}(x)\right) \geq \frac{1}{2} f^{\circ\left(N_{n}\right)}(x) \rightarrow \infty$.

Takens proceeds in two steps.

(1) He shows that $f$ is conjugate to a diffeomorphism $f_{1}(x)=x-x^{p+1}+\alpha x^{2 p+1}+g_{1}(x)$, where $g_{1}$ is a $C^{\infty}$ function flat at 0 . We can see this at once from Corollary 3.2. The Taylor series of $f$ is conjugate to $X-X^{p+1}+\alpha X^{2 p+1}$, so choose an invertible series $H$ that has

$$
H^{\circ-1} T_{0} f H=X-X^{p+1}+\alpha X^{2 p+1} .
$$

Then choose $h \in \operatorname{Diffeo}(\mathbb{R})$ i, fixing 0 , with $T_{0} h=H$, and let $f_{1}=f^{h}$.

(2) He defines $\Psi: \mathbb{R}^{2} \rightarrow \mathbb{R}^{2}$ by

$$
\Psi(x, t)=\left(x-x^{p+1}+\alpha x^{2 p+1}+t g_{1}(x), t\right)
$$

and shows that there is a vector field

$$
\widetilde{Z}=Z(x, t) \frac{\partial}{\partial x}+\frac{\partial}{\partial t}
$$

with $Z$ flat at all points where $x=0$, and $\Psi_{*}(\widetilde{Z})=\widetilde{Z}$ on a neighborhood of the segment $\{(x, t): x=0,0 \leq t \leq 1\}$. The proof of the existence of such a vector field (which is a fixed-point theorem) requires a substantial argument [30, pp. 177-189], and we omit the details. Once he has it, the conjugacy of $f_{1}$ to $g$ is obtained by taking $\phi(x)$ so that, for small $x>0$, the points $(x, 1)$ and $(\phi(x), 0)$ lie on the same integral curve of $\widetilde{Z}$.

Ahern and Rosay [2, pp. 549-551] give another proof of his theorem. They show that, in fact, if Condition ( $\mathrm{T}$ ) holds, then the "basic trick" construction of Theorem 2.2. condition (4) may be used, with caution, on a neighborhood of 0 , to get a conjugacy started.

\section{$\S 5 .[0,+\infty)$ : The PRODUCT CONDITION}

Fix arbitrary $f, g \in S_{-}$.

Lemma 5.1. Suppose $f$ and $g$ are conjugate in $\operatorname{Diffeo}([0, \infty))$. Then for any $x>0$ there exists $\xi>0$ such that the product (1) converges.

Proof. Pick $h \in \operatorname{Diffeo}([0, \infty))$ with $f=g^{h}$, and set $\xi=h(x)$. We observe that $h \circ f^{\circ n}=$ $g^{\circ n} \circ h$. Hence equating derivatives we get

$$
h^{\prime}\left(f^{\circ n}(x)\right) \frac{d f^{\circ n}}{d x}(x)=\frac{d g^{\circ n}}{d \xi}(\xi) h^{\prime}(x)
$$

hence

$$
\prod_{j=0}^{n-1} \frac{f^{\prime}\left(f^{\circ j}(x)\right)}{g^{\prime}\left(g^{\circ j}(\xi)\right)}=\frac{h^{\prime}(x)}{h^{\prime}\left(f^{\circ n}(x)\right)},
$$

so the product converges to the limit $h^{\prime}(x) / h^{\prime}(0)$.

The correspondence between $x$ and $\xi$, referred to in the lemma, is not essential, for we have the following, which is due to Kopell [10]. (We give the proof for convenience.)

Lemma 5.2. Let $x, y \in[0, \infty)$ and denote $x_{n}=f^{\circ n}(x), y_{n}=f^{\circ n}(y)$. Then the infinite product

$$
\prod_{n=0}^{\infty} \frac{f^{\prime}\left(x_{n}\right)}{f^{\prime}\left(y_{n}\right)}
$$

converges. 
Proof. First, assume that $y_{0}$ is between $x_{1}$ and $x_{0}$. The convergence of the product is equivalent to the convergence of the series of logarithms $\sum_{n=0}^{\infty} \ln \left(\frac{f^{\prime}\left(x_{n}\right)}{f^{\prime}\left(y_{n}\right)}\right)$, which in turn is equivalent to that of $\sum_{n=0}^{\infty}\left(1-\frac{f^{\prime}\left(x_{n}\right)}{f^{\prime}\left(y_{n}\right)}\right)$. Now

$$
\left|\frac{f^{\prime}\left(x_{n}\right)-f^{\prime}\left(y_{n}\right)}{f^{\prime}\left(y_{n}\right)}\right| \leq\left(\frac{\sup \left|f^{\prime \prime}\right|}{\inf \left|f^{\prime}\right|}\right) \cdot\left|x_{n}-y_{n}\right|
$$

(where the sup and inf are taken on $[0, x]$; note that the inf is positive since $f$ is a diffeomorphism), and so the convergence follows from $\sum_{n=0}^{\infty}\left|x_{n}-y_{n}\right| \leq\left|x_{0}\right|$, which holds because the intervals from $x_{n}$ to $y_{n}$ are pairwise-disjoint subintervals of that from 0 to $x_{0}$.

So the result holds when $y_{0}$ is between $x_{1}$ and $x_{0}$.

For general $y$, choose $k \in \mathbb{Z}$ such that $y_{0}$ is between $x_{k}$ and $x_{k+1}$. Then

$$
\prod_{n=0}^{m} \frac{f^{\prime}\left(x_{n}\right)}{f^{\prime}\left(y_{n}\right)}=\frac{f^{\prime}\left(x_{0}\right) \cdots f^{\prime}\left(x_{k-1}\right)}{f^{\prime}\left(y_{m-k+1}\right) \cdots f^{\prime}\left(y_{m}\right)} \cdot \prod_{n=0}^{m-k} \frac{f^{\prime}\left(x_{n+k}\right)}{f^{\prime}\left(y_{n}\right)},
$$

so the series

$$
\prod_{n=0}^{\infty} \frac{f^{\prime}\left(x_{n}\right)}{f^{\prime}\left(y_{n}\right)} \text { and } \prod_{n=0}^{\infty} \frac{f^{\prime}\left(x_{n+k}\right)}{f^{\prime}\left(y_{n}\right)}
$$

converge or diverge together, with

$$
\prod_{n=0}^{\infty} \frac{f^{\prime}\left(x_{n}\right)}{f^{\prime}\left(y_{n}\right)}=\frac{f^{\prime}\left(x_{0}\right) \cdots f^{\prime}\left(x_{k-1}\right)}{f^{\prime}(0)^{k}} \cdot \prod_{n=0}^{\infty} \frac{f^{\prime}\left(x_{n+k}\right)}{f^{\prime}\left(y_{n}\right)} .
$$

Thus we obtain the general result by replacing $x_{n}$ by $x_{n+k}$.

Corollary 5.3. (1) In case $x_{1}<y<x_{0}$, and $T_{0} f=X+b X^{p+1}+\cdots$ for some $p \in \mathbb{N}$, the product (8) is $1+O\left(x^{p}\right)$ as $x \downarrow 0$, uniformly for $y$ between $x_{1}$ and $x_{0}$.

(2) In case $T_{0} f=X$ the product is $1+o\left(x^{n}\right)$, for any $n$.

Proof. (1) Just use the estimate $f^{\prime \prime}(x)=O\left(x^{p-1}\right)$.

(2) follows from (1).

Corollary 5.4. If the product (11) converges for some $x, \xi>0$, then it converges for any choice of $x, \xi>0$.

Corollary 5.5. Suppose $f$ and $g$ are conjugate in $\operatorname{Diffeo}([0, \infty))$. Then for any $x>0$ and $\xi>0$ the product (11) converges.

Corollary 5.6. The convergence or divergence of the product (11) is not affected if the functions $f$ and $g$ are replaced by conjugates.

Condition $(\mathrm{P})$ is actually a consequence of Condition $(\mathrm{T})$ in the nonflat cases.

Proposition 5.7. (1) If $f^{\prime}(0) \neq 1$ or $g^{\prime}(0) \neq 1$, then Condition (P) is equivalent to $f^{\prime}(0)=g^{\prime}(0)$.

(2) If $f$ and $g$ have conjugate nonidentity Taylor series, then Condition $(\mathrm{P})$ is satisfied.

Proof. (1) To prove the first assertion, assume that $f^{\prime}(0)=\alpha<1$. Choose $\alpha^{\prime}$ with $\alpha<\alpha^{\prime}<1$. Then, for sufficiently small $x$ we have $f(x)<\alpha^{\prime} x$. Consider the product $\prod_{n=0}^{\infty} \frac{f^{\prime}\left(f^{\circ n}(x)\right)}{\alpha}$. The product converges if and only if $\sum_{n=0}^{\infty}\left(1-\frac{f^{\prime}\left(f^{\circ n}(x)\right)}{\alpha}\right)$ converges. But the second derivative of $f$ is bounded near 0 , so the sum is dominated by a constant times $\sum_{n=0}^{\infty} f^{\circ n}(x) \leq \sum_{n=0}^{\infty}\left(\alpha^{\prime}\right)^{n}<\infty$, and hence is indeed convergent.

Now consider the similar product for $g$. The product in Condition $(\mathrm{P})$ is the quotient of the products if $f^{\prime}(0)=g^{\prime}(0)$, and hence converges as well, and (for the same reason) it does not converge (to a nonzero limit) if $f^{\prime}(0) \neq g^{\prime}(0)$. 
(2) By replacing $g$ with a conjugate which has the same Taylor series as $f$ we reduce to the case in which $f$ and $g$ have coincident Taylor series. The result then follows from the next, more general lemma, which we will also use again later.

Lemma 5.8. Let $T_{0}(f)=T_{0}(g)=X+b X^{p+1}+\cdots\left(\bmod X^{2 p+1}\right)$, where $p \in \mathbb{N}$ and $b \neq 0$. Then

$$
\prod_{n=0}^{\infty} \frac{f^{\prime}\left(f^{\circ n}(x)\right)}{g^{\prime}\left(g^{\circ n}(x)\right)}=1+O\left(x^{p}\right) .
$$

Proof. Without loss in generality, we take $b<0$, and write $c=-b$. We use $C$ for a positive constant that may differ at each occurrence. We may assume that the $x>0$ under consideration are so small, that $\left|f(x)-x+c x^{p+1}\right| \leq C x^{p+2}$ and $|C x|<\frac{1}{2} c$. This means that $c x_{n}^{p+1}-C x_{n}^{p+2}<x_{n}-x_{n+1}<c x_{n}^{p+1}+C x_{n}^{p+2}$, where $x_{n}=f^{\circ n}(x)$. So, for $0<\alpha<1$ between $x$ and $\alpha x$ there are no more than

$$
\frac{(1-\alpha) x}{\left(c(\alpha x)^{p+1}-C x^{p+2}\right)}=\frac{(1-\alpha) \alpha^{-(p+1)}}{c x^{p}\left(1-\alpha^{-(p+1)} C x\right)}
$$

and no fewer than

$$
\frac{(1-\alpha) x}{\left(c x^{p+1}+C x^{p+2}\right)}=\frac{1-\alpha}{c x^{p}(1+C x)}
$$

points from the $f$-orbit of $x$.

Let us start by reformulating the claim: It is enough to prove that

$$
\log \left(\prod_{n=0}^{\infty} \frac{f^{\prime}\left(f^{\circ n}(x)\right)}{g^{\prime}\left(g^{\circ n}(x)\right)}\right)=\sum_{n=0}^{\infty} \log \left(\frac{f^{\prime}\left(f^{\circ n}(x)\right)}{g^{\prime}\left(g^{\circ n}(x)\right)}\right)=O\left(x^{p}\right) .
$$

As $f^{\prime}(0)=g^{\prime}(0)=1$ and $|\log (t)| \sim|1-t|$ is close to $t=1$, it is enough to prove that

$$
\sum_{n=0}^{\infty}\left|g^{\prime}\left(g^{\circ n}(x)\right)-f^{\prime}\left(f^{\circ n}(x)\right)\right|=O\left(x^{p}\right) .
$$

Since $T_{0} f=T_{0} g$, we may also assume that $x$ is so small that $\left|f^{\prime}(x)-g^{\prime}(x)\right|<C x^{2 p}$. We then observe that, since $\left|x_{n}-x_{n+1}\right|>(c / 2) x_{n}^{p+1}$, we have the estimate

$$
\sum_{k=0}^{\infty} x_{k}^{p+1} \leq \frac{2}{c} \sum_{k=0}^{\infty}\left|x_{k}-x_{k+1}\right|=\frac{2 x_{0}}{c} .
$$

As $\left|f^{\prime}(x)-g^{\prime}(x)\right|<C x^{2 p}$ for all $x$ in question, we have

$$
\sum_{k=0}^{\infty}\left|f^{\prime}\left(x_{k}\right)-g^{\prime}\left(x_{k}\right)\right| \leq C \sum_{k=0}^{\infty} x_{k}^{2 p} \leq C x_{0}^{p-1} \sum_{k=0}^{\infty} x_{k}^{p+1}=O\left(x_{0}^{p}\right),
$$

and the estimate can be reduced to estimating $\sum\left|g^{\prime}\left(x_{n}\right)-g^{\prime}\left(g^{\circ n}(x)\right)\right|$. Since $g^{\prime \prime}=$ $O\left(x^{p-1}\right)$ we have $\left|g^{\prime}(r)-g^{\prime}(s)\right| \leq O\left(s^{p-1}\right)|r-s|$ for $r<s$, and it remains to show that $\sum\left|f^{\circ n}(x)-g^{\text {on }}(x)\right|<C x$.

Let us now consider only points so close to the origin that $|f(x)-g(x)|<C x^{2 p+1}$. For those points we have the estimate

$$
\begin{aligned}
\left|f^{\circ n}(x)-g^{\circ n}(x)\right| & \leq\left|f^{\circ n}(x)-f\left(g^{\circ(n-1)}(x)\right)\right|+\left|f\left(g^{\circ(n-1)}\right)(x)-g^{\circ n}(x)\right| \\
& \leq M_{x}\left|f^{\circ(n-1)}(x)-g^{\circ(n-1)}(x)\right|+C\left(g^{\circ(n-1)}(x)\right)^{2 p+1} \\
& \leq C\left(M_{x}^{n}+\cdots+1\right) x^{2 p+1},
\end{aligned}
$$

where $M_{x}$ is the maximum of $f^{\prime}$ on the interval $[0, x]$ and thus can (for small $x$ ) be estimated from above by 1 (since $b<0$ ). This gives us $\left|f^{\circ n}(x)-g^{\circ n}(x)\right| \leq C n x^{2 p+1}$. 
Let us consider the first point in the orbit of $x$ with respect to $f$ which is less than $\alpha x$. Let it be $f^{\circ n_{1}}(x)$. Then by the observation at the beginning of the proof, for $\alpha>\frac{1}{2}$, and $x$ sufficiently small, $n_{1}<(1-\alpha) C / x^{p}$, where the constant depends only on the Taylor expansion. By the previous paragraph, for any $k \leq n_{1}$,

$$
\left|f^{\circ k}(x)-g^{\circ k}(x)\right| \leq \frac{(1-\alpha) C x^{2 p+1}}{x^{p}}=(1-\alpha) C x^{p+1} .
$$

As $|f(y)-y|>\frac{c}{2} y^{p+1}$, we see that for a choice of $\alpha<1$ sufficiently close to 1 , we have $\frac{1}{2}\left(f^{\circ(k+1)}(x)+f^{\circ k}(x)\right)<g^{\circ k}(x)<\frac{1}{2}\left(f^{\circ k}(x)+f^{\circ(k-1)}(x)\right)$. Thus the intervals $\left[f^{\circ k}(x), g^{\circ k}(x)\right]$ are disjoint and $\sum_{k=0}^{n_{1}}\left|f^{\circ k}(x)-g^{\circ k}(x)\right| \leq(1-\alpha) x+C x^{p+1}$. On the other hand, in the particular case, $k=n_{1}$, if $g^{\circ\left(n_{1}+1\right)}(x)=x^{(1)}$, we have $\sum_{m=0}^{\infty}\left|f^{\circ\left(n_{1}+1+m\right)}(x)-f^{\circ m}\left(x^{(1)}\right)\right| \leq \alpha x$, as the sum of lengths of disjoint intervals. This means that

$$
\begin{aligned}
\sum_{n=0}^{\infty}\left|f^{\circ n}(x)-g^{\circ n}(x)\right| \leq & \sum_{n=0}^{n_{1}}\left|f^{\circ n}(x)-g^{\circ n}(x)\right|+\sum_{m=0}^{\infty}\left|f^{\circ m}\left(x^{(1)}\right)-g^{\circ m}\left(x^{(1)}\right)\right| \\
& +\sum_{m=0}^{\infty}\left|f^{\circ\left(n_{1}+1+m\right)}(x)-f^{\circ m}\left(x^{(1)}\right)\right|+C x^{p+1} \\
\leq & (1-\alpha) x+\sum_{m=0}^{\infty}\left|f^{\circ m}\left(x^{(1)}\right)-g^{\circ m}\left(x^{(1)}\right)\right|+\alpha x+C x^{p+1} \\
\leq & 2 x+\sum_{m=0}^{\infty}\left|f^{\circ m}\left(x^{(1)}\right)-g^{\circ m}\left(x^{(1)}\right)\right| .
\end{aligned}
$$

Using this argument inductively we deduce that

$$
\sum_{n=0}^{\infty}\left|f^{\circ n}(x)-g^{\circ n}(x)\right| \leq 2 x+2 x^{(1)}+\cdots \leq 2 \sum_{j=0}^{\infty} \alpha^{j} x=C x
$$

and we are done.

Example 5.1. Notice that for the particular case $p=1$ this lemma says that the Condition (P) is satisfied for $f(x)=x+x^{2}$ and $g(x)=x+x^{2}+x^{3}$. On the other hand, the Taylor series $X+X^{2}$ and $X+X^{2}+X^{3}$ are not conjugate, which shows that the Condition $(\mathrm{P})$ is strictly weaker than Condition $(\mathrm{T})$ in the nonflat case.

We shall see shortly that Condition $(\mathrm{P})$ guarantees the existence of a $C^{1}$ diffeomorphism conjugating $f$ to $g$. Thus the existence of a $C^{1}$ conjugacy is strictly weaker than the existence of a $C^{\infty}$ conjugacy.

We mention here the observations of Young 34. He considered $C^{2}$ diffeomorphisms $f$ on $[0,+\infty)$ with $T_{0} f=x+a x^{2}\left(\bmod x^{3}\right)$, and with $a \neq 0$. A result of Szekeres (cf. [13, Theorem 8.4.5]) implies that all such $C^{2}$ diffeomorphisms (having $a$ of one sign) are $C^{1}$-conjugate. Young showed that they are in fact $C^{2}$-conjugate.

\section{$\S 6 .[0,+\infty)$ : Sufficient CONDitions}

6.1. The differential equation. Suppose $f, g \in \operatorname{Diffeo}([0,+\infty))$ fix only 0 , both belong to $S_{-}$and satisfy Condition $(\mathrm{P})$.

We define

$$
F_{1 a}(x)=H_{1}(f, f ; x, a)=\prod_{n=0}^{\infty} \frac{f^{\prime}\left(f^{\circ n}(x)\right)}{f^{\prime}\left(f^{\circ n}(a)\right)}
$$


whenever $a, x>0$. Note that

We define

$$
F_{1 a}(x)=\lim _{n \uparrow \infty} \frac{\left(f^{\circ n}\right)^{\prime}(x)}{\left(f^{\circ n}\right)^{\prime}(a)} .
$$

whenever $\alpha, \xi>0$.

$$
G_{1 \alpha}(\xi)=H_{1}(g, g ; \xi, \alpha)=\prod_{n=0}^{\infty} \frac{g^{\prime}\left(g^{\circ n}(\xi)\right)}{g^{\prime}\left(g^{\circ n}(\alpha)\right)}
$$

Lemma 6.1. Fix $a>0, \alpha>0$. The functions $x \mapsto F_{1 a}(x)$ and $\xi \mapsto G_{1 \alpha}(\xi)$ are infinitely differentiable and positive on $(0+\infty)$, and hence

$$
(x, \xi) \mapsto H_{1}(x, y)=H_{1}(a, \alpha) F_{1 a}(x) / G_{1 \alpha}(\xi)
$$

is infinitely differentiable and positive on $(0,+\infty) \times(0,+\infty)$.

Proof. It suffices to show that $x \mapsto F_{1 a}(x)$ is infinitely differentiable on $(0,+\infty)$ for each $a>0$. The argument for $\xi \mapsto G_{1 \alpha}(\xi)$ is precisely analogous.

Fix $a \in(0,+\infty)$. Let $J_{a}$ denote the closed interval from 0 to $a$. Let $a_{n}=f^{\circ n}(a)$, for all $n \in \mathbb{Z}$. Let $I_{a}$ denote the closed interval from $a_{1}$ to $a$. Let

$$
D_{j}=\max _{J_{a}}\left|f^{(j)}\right|, \quad \forall j \in \mathbb{Z} .
$$

(Note that $\min _{J_{a}}\left|f^{\prime}\right|=\left(D_{-1}\right)^{-1}$.)

For $x \in(0,+\infty)$, let $x_{n}=f^{\circ n}(x)$, for all $n \in \mathbb{Z}$. For ease of notation, we abbreviate $\frac{d}{d x} f^{\circ n}(x)=f^{\prime}(x) f^{\prime}\left(x_{1}\right) \cdots f^{\prime}\left(x_{n-1}\right)$ to $x_{n}^{\prime}$, and similarly denote $\frac{d^{k}}{d x^{k}} f^{\circ n}(x)$ by $x_{n}^{(k)}$. We use $x_{n}^{\prime \prime}$ for $x_{n}^{(2)}$, etc.

Before continuing the proof, we pause to note a couple of lemmas that follow from Lemma 5.2 .

In what follows, unless otherwise specified, we use $K$ to denote a constant that depends at most on $f$, and $a$, and that may be different at each occurrence.

\section{Lemma 6.2.}

whenever $x \in I_{a}$.

$$
K^{-1}\left|\left(f^{\circ n}\right)^{\prime}(a)\right| \leq\left|x_{n}^{\prime}\right| \leq K\left|\left(f^{\circ n}\right)^{\prime}(a)\right|
$$

Proof. We have

$$
\frac{\left(f^{\circ n}\right)^{\prime}(a)}{\left(f^{\circ n}\right)^{\prime}(x)}=\prod_{j=0}^{n-1} \frac{f^{\prime}\left(a_{j}\right)}{f^{\prime}\left(x_{j}\right)},
$$

so the result follows from the uniform convergence of $\prod_{j=0}^{\infty} \frac{f^{\prime}\left(a_{j}\right)}{f^{\prime}\left(x_{j}\right)}$, for $x \in I_{a}$.

Lemma 6.3.

whenever $x \in I_{a}$.

$$
\left|x_{n}^{\prime}\right| \leq K\left|\frac{x_{n+1}-x_{n}}{x_{1}-x_{0}}\right|
$$

Proof. By the Law of the Mean,

$$
\frac{x_{n+1}-x_{n}}{x_{1}-x_{0}}=\left(f^{\circ n}\right)^{\prime}(y)
$$

for some $y$ between $x$ and $x_{1}$, so the result follows from a few applications of the previous lemma.

Lemma 6.4. $\left|x_{1}-x\right| \geq K\left|a_{1}-a\right|$, for all $x \in I_{a}$. 
Proof. For $x \in I_{a}, f(a) \leq x \leq a$, so $f(x) \leq f(a) \leq x$, so $|f(x)-x|=|f(x)-f(a)|+$ $|x-f(a)| \geq\left(D_{-1}\right)^{-1}|x-a|+\left|x-a_{1}\right| \geq \min \left\{1,\left(D_{-1}\right)^{-1}\right\}\left|a-a_{1}\right|$.

Proof of Lemma 6.1. It suffices to show that the logarithm

$$
\log F_{1 a}(x, \xi)=\sum_{n=0}^{+\infty}\left\{\log f^{\prime}\left(x_{n}\right)-\log f^{\prime}\left(a_{n}\right)\right\}
$$

is infinitely differentiable.

The term by term derivative with respect to $x$ is the series

$$
\sum_{n=0}^{+\infty} \frac{f^{\prime \prime}\left(x_{n}\right) x_{n}^{\prime}}{f^{\prime}\left(x_{n}\right)}
$$

and it will be convenient to denote the $n$th term by

$$
T_{n}(x)=\frac{f^{\prime \prime}\left(x_{n}\right) x_{n}^{\prime}}{f^{\prime}\left(x_{n}\right)}
$$

and the $n$th partial sum by

$$
S_{n}(x)=\sum_{j=0}^{n-1} T_{j}(x) .
$$

It will suffice to show that for each nonnegative integer $k, S_{n}^{(k)}(x)$ converges uniformly on $I_{a}$.

For any smooth function $\rho:(0,+\infty) \rightarrow(0,+\infty)$, and $k \in \mathbb{N}$, let us define $A_{k}(\rho)$ as the function

$$
A_{k}(\rho)=\frac{d^{k}}{d x^{k}}\left(\frac{f^{\prime \prime}(\rho) \rho^{\prime}}{f^{\prime}(\rho)}\right)-\frac{f^{\prime \prime}(\rho) \rho^{(k+1)}}{f^{\prime}(\rho)} .
$$

Then a straightforward induction establishes that $A_{k}(\rho)(x)$ is the sum of $M_{k}$ terms (where the integer $M_{k}$ depends on $k$, but not on $\rho$ ), each of which is a finite product

$$
\frac{\gamma \prod_{i} f^{\left(r_{j}\right)}(\rho(x)) \prod_{j} \rho^{\left(t_{j}\right)}(x)}{\left(f^{\prime}(\rho(x))\right)^{k+1}}
$$

where the coefficients $\gamma$ are fixed integers independent of $f$, where each $r_{i} \leq k+2$, each $t_{j} \leq k$, and at least one $t_{j}$ is present.

The term $T_{n}^{(k)}$ takes the form

$$
A_{k}\left(x_{n}\right)+\frac{f^{\prime \prime}\left(x_{n}\right) x_{n}^{(k+1)}}{f^{\prime}\left(x_{n}\right)} .
$$

To begin with, we observe that by the last two lemmas,

$$
\left|\frac{f^{\prime \prime}\left(x_{n}\right) x_{n}^{\prime}}{f^{\prime}\left(x_{n}\right)}\right| \leq K D_{2} D_{-1}\left|x_{n+1}-x_{n}\right|, \quad \forall x \in I_{a} ;
$$

hence $\left\{S_{n}(x)\right\}$ itself converges uniformly on $I_{a}$, with the error in $S_{n}(x)$ bounded by $K D_{2} D_{-1} a_{n}$, where $a_{n}=f^{\circ n}(a)$.

Now we will proceed by induction on $k$, and we first consider the first derivatives $T_{n}^{\prime}(x)$ and note that

where

$$
T_{n}^{\prime}(x)=A_{1}\left(x_{n}\right)+B_{1}\left(x_{n}\right),
$$

and

$$
A_{1}\left(x_{n}\right)=\left\{\frac{f^{\prime \prime \prime}\left(x_{n}\right)\left(x_{n}^{\prime}\right)^{2}}{f^{\prime}\left(x_{n}\right)}-\frac{\left(f^{\prime \prime}\left(x_{n}\right) x_{n}^{\prime}\right)^{2}}{\left(f^{\prime}\left(x_{n}\right)\right)^{2}}\right\}
$$

$$
B_{1}\left(x_{n}\right)=\frac{f^{\prime \prime}\left(x_{n}\right) x_{n}^{\prime \prime}}{f^{\prime}\left(x_{n}\right)}
$$


Estimating each of its terms by its maximum, we see that $A_{1}\left(x_{n}\right)$ is dominated by

$$
K^{2}\left(D_{3} D_{-1}+D_{2}^{2} D_{-1}^{2}\right)\left|x_{n+1}-x_{n}\right| \leq K\left|x_{n+1}-x_{n}\right|,
$$

for a (different) constant $K$.

A calculation yields $x_{n}^{\prime \prime}=x_{n}^{\prime} S_{n}$, so the term $B_{1}\left(x_{n}\right)$ is dominated by

$$
K^{2} D_{2} D_{-1}\left|x_{n+1}-x_{n}\right|,
$$

and we conclude that $S_{n}^{\prime}(x)$ also converges uniformly on $I_{a}$, with error bounded by $K x_{n}$.

We also observe that $\left|S_{n}^{\prime}(x)\right| \leq K x$.

Now we formulate an induction hypothesis $P_{k}$ :

There exist a constant $K$, depending only on $f, a$, and $k$, such that

(a) for $0 \leq j \leq k-1$ and each $n \geq 0$,

$$
\left|T_{n}^{(j)}(x)\right| \leq K\left|x_{n+1}-x_{n}\right| \quad \text { and } \quad\left|S_{n}^{(j)}(x)\right| \leq K, \quad \text { and }
$$

(b) for $1 \leq j \leq k$,

$$
\left|x_{n}^{(j)}\right| \leq K\left|x_{n+1}-x_{n}\right|
$$

We have established $P_{2}$.

Suppose $P_{k}$ holds, for some $k \geq 2$. Differentiating the formula $x_{n}^{\prime \prime}=x_{n}^{\prime} S_{n} k-1$ times we get

$$
x_{n}^{(k+1)}=\sum_{j=0}^{k-1}\left(\begin{array}{c}
k-1 \\
j
\end{array}\right) x_{n}^{(j+1)} S_{n}^{(k-j-1)},
$$

so conditions (a) and (b) of the hypothesis yield

$$
\left|x_{n}^{(k+1)}\right| \leq \sum_{j=0}^{k-1}\left(\begin{array}{c}
k-1 \\
j
\end{array}\right) K\left|x_{n+1}-x_{n}\right|=K\left|x_{n+1}-x_{n}\right|
$$

(with a new $K$ ), and condition (b) of $P_{k+1}$ is proven.

Condition (a) then follows because of the form of $T_{n}^{(k)}$.

Thus, by induction, $P_{k}$ holds for each $k \geq 2$.

Thus $S_{n}^{(k)}=\sum T_{m}^{(k)}$ converges uniformly for all $k$, and Lemma 6.1 is proved. 2) ).

We now consider the three-parameter initial-value problem $D_{1}(a, \alpha, \lambda)$ (cf. equation

It follows from Lemma 6.1 and standard results about ordinary differential equations [4, p. 22] that problem $D_{1}(a, \alpha, \lambda)$ has a unique infinitely differentiable solution $\phi(a, \alpha, \lambda ; x)$ near $x=a$ whenever (P) holds, $a, \alpha>0$, and $\lambda>0$. Obviously, the solution is a strictly increasing function of $x$ and its domain is an open subinterval of $(0,+\infty)$, containing $a$.

Note that

$$
\prod_{j=n}^{m} f^{\prime}\left(x_{j}\right)=\left(f^{\circ(m-n)}\right)^{\prime}\left(x_{n}\right) \approx \frac{x_{m+1}-x_{m}}{x_{n+1}-x_{n}},
$$

so the product tends to 0 as $m \rightarrow+\infty$. It follows that the product $H_{1}(x, y)$ does not extend continuously to the closed quadrant $[0,+\infty) \times[0,+\infty)$, nor even to the corner $(0,0)$, so there is no point in considering the differential equations at the endpoint. In fact, a moment's thought reveals that $H_{1}(x, y)$ tends to $\infty$ as $x \rightarrow 0$ for fixed $y>0$, and tends to 0 as $y \rightarrow 0$ for fixed $x>0$, so all positive numbers may be obtained as limits of $H_{1}(x, y)$ for a suitable approach to $(0,0)$ from inside $J \times J$.

Lemma 6.5. Assume $f, g \in S_{-}$and Condition (P) holds. Then for each $a, \alpha>0$ and each $\lambda>0$ the domain of the solution to problem $D_{1}(a, \alpha, \lambda)$ is $(0,+\infty)$. 
Proof. The domain $U$ of the solution $\phi$ is a nonempty connected subset of $J=(0,+\infty)$, and one sees easily that it is open. In fact, if either end (say $c$ ) of $U$ lies inside $J$, then since $\phi^{\prime}$ is bounded on $(a, c), \phi$ has a continuous extension to $c$, and by uniqueness $\phi$ is the solution to $D_{1}(c, \phi(c), \lambda)$, so $\phi$ extends to a neighborhood of $c$, a contradiction. Thus $U=J$, and $\phi$ conjugates $f$ to $g$ on the whole of $J$.

The following lemma reformulates the information in the proof of Lemma 5.1]in other language.

Lemma 6.6. Suppose $f, g, h \in \operatorname{Diffeo}([0,+\infty))$ and $f=g^{h}$. Then $\phi=h$ is the solution to problem $D_{1}\left(a, h(a), h^{\prime}(0)\right)$, whenever $a>0$.

Not all solutions to the initial-value problems $D_{1}(a, \alpha, \lambda)$ will be conjugating maps. For a start, we would need to ensure the condition $\phi(f(a))=g(\alpha)$. This leads us to the following.

Lemma 6.7. Assume Condition (P), with $f, g \in S_{-}$. Then for each $a>0$, and each $\alpha>0$, there exists $\lambda>0$ such that the solution $\phi(a, \alpha, \lambda)$ to problem $D_{1}(a, \alpha, \lambda)$ has $\phi(f(a))=g(\alpha)$.

Proof. Given $a$ and $\alpha$, we could start by trying $\lambda=1$. If the solution $\phi_{1}$ to $D_{1}(a, \alpha, 1)$ has $\phi_{1}(f(a))=g(\alpha)$, we take $\lambda=1$ and are done. If $\phi_{1}(f(a))<g(\alpha)$, then decreasing $\lambda$ eventually reduces $\phi^{\prime}$ to very small values on the interval $[a, f(a)]$, and hence pulls $\phi(f(a))$ above $g(\alpha)$. Thus, since $\phi(f(a))$ varies continuously with $\lambda$, there exists some $\lambda$ with $\phi(a, \alpha, \lambda)(f(a))=g(\alpha)$, and we are done. If $\phi_{1}(f(a))>g(\alpha)$, then we can attain a similar result by increasing $\lambda$ instead, because this increases $\phi^{\prime}$ to very large values. More precisely, $H_{1}(x, y)$ is bounded below by a positive constant, say $\kappa$, on $[f(a), a] \times[g(\alpha), \alpha]$, so if $\phi(f(a)) \geq g(\alpha)$, then $\phi^{\prime}>\kappa \lambda$ on $[f(a), a]$; hence

$$
\alpha-g(\alpha) \geq \phi(a)-\phi(f(a)) \geq \lambda \kappa(a-f(a)),
$$

which is impossible for large $\lambda$. Thus for large enough $\lambda$, we have $\phi(f(a))<g(\alpha)$, so another application of the intermediate value theorem tells us that there exists some $\lambda$ with $\phi(a, \alpha, \lambda)(f(a))=g(\alpha)$.

Now we proceed to show that the solution $\phi$ of Lemma (6.7) conjugates $f$ to $g$ on $(0,+\infty)$.

Lemma 6.8. Suppose that $u$ is a differentiable real-valued function on an open interval $U$, and for some constant $\kappa>0$ we have

$$
\left|u^{\prime}(x)\right| \leq \kappa \cdot|u(x)|, \quad \forall x \in U .
$$

Suppose that $u$ has a zero in $U$. Then $u$ is identically zero on $U$.

Proof. The set $Z=u^{\circ-1}(0)$ of zeros of $u$ in $U$ is relatively closed, and nonempty, so it suffices to show that it is open. Fix $a \in Z$, and choose $\epsilon>0$ so that $a \pm \epsilon \in U$ and $\epsilon \kappa<1$. Let $M$ be the maximum of $|u|$ on the closed interval $J=[a-\epsilon, a+\epsilon]$.

If $M>0$, then choose $b \in J$ with $|u(b)|=M$. By the Law of the Mean, we may choose $c$ between $a$ and $b$ with $|u(b)|=\left|u^{\prime}(c)\right| \cdot|b-a|$. But then

$$
M=|u(b)| \leq \kappa M \cdot \epsilon<M,
$$

which is impossible.

Thus $M=0$, so $a$ is an interior point of $Z$.

Thus $Z$ is open, and we are done. 
Lemma 6.9. Suppose (P). Fix $a, \alpha>0$. Choose $\lambda>0$ such that the solution $h(x)=$ $\phi(a, \alpha, \lambda ; x)$ to problem $D_{1}(a, \alpha, \lambda)$ has $h(f(a))=g(\alpha)$. Then the domain of the solution is $J=(0,+\infty), h$ maps $J$ onto $J$ and $g \circ h=h \circ f$ on $J$.

Proof. We establish that on each compact subinterval of $J$ we have an inequality $\left|u^{\prime}\right| \leq$ $\kappa \cdot|u|$, where

$$
u(x)=g(\phi(x))-\phi(f(x)) .
$$

In detail, one calculates (by fiddling with products) that

$$
u^{\prime}(x)=\lambda\left\{H_{1}(x, \phi(x)) \cdot g^{\prime}(\phi(x))-H_{1}\left(x, g^{-1}(\phi(f(x)))\right) \cdot g^{\prime}\left(g^{-1}(\phi(f(x)))\right)\right\},
$$

and (using the Law of the Mean) estimates this (on a compact subinterval of $J$ ) by

$$
\kappa_{1} \times\left|g^{-1}(\phi(f(x)))-\phi(x)\right| \leq \kappa_{2}|\phi(f(x))-g(\phi(x))|=\kappa_{2}|u(x)| .
$$

Then we apply Lemma 6.8 and the fact that $u(a)=g(\alpha)-\phi(f(a))=0$. This tells us that $u(x)=0$ on the domain of $\phi$, which is $J$.

These results tell us that the initial-value problem together with the conjugation equation at one point are enough to guarantee the conjugation equation on the whole interval $J=(0,+\infty)$.

Lemma 6.10. Suppose Condition $(\mathrm{P})$ holds. If $\phi:[0,+\infty) \rightarrow[0,+\infty)$ satisfies

$$
\left.\begin{array}{rl}
\phi(f(x)) & =g(\phi(x)), \\
\phi^{\prime}(x) & =H_{1}(x, \phi(x)) \lambda
\end{array}\right\} \quad \forall x \in J
$$

then $\lim _{x \rightarrow d} \phi^{\prime}(x)=\lambda$ and $\phi$ has a one-sided derivative at $d$, equal to $\lambda$.

Proof. Fix some $a \in J$ and denote $I_{a}=[f(a), a]$.

For fixed $x \in I_{a}$, letting $x_{n}=f^{\circ n}(x)$, we have

$$
\begin{aligned}
\phi\left(f^{\circ n}(x)\right) & =g^{\circ n}(\phi(x)), \\
\phi^{\prime}\left(x_{n}\right) \cdot x_{n}^{\prime} & =\left(g^{\circ n}\right)^{\prime}(\phi(x)) \cdot \phi^{\prime}(x), \\
\phi^{\prime}\left(x_{n}\right) & =\prod_{j=0}^{n-1}\left(\frac{g^{\prime}\left(g^{\circ j}(\phi(x))\right)}{f^{\prime}\left(x_{j}\right)}\right) \cdot \phi^{\prime}(x) .
\end{aligned}
$$

Since the product converges to $H_{1}(x, \phi(x))^{-1}$, the right-hand side converges to $\lambda$, so the derivative $\phi^{\prime}$ extends continuously from $J$ to 0 if $\phi$ is given the value 0 there. This is enough to force the rest of the conclusions.

Finally, we show that the $\lambda$ is unique.

Lemma 6.11. Suppose Condition (P) holds. Then, for each given a, $\alpha \in J$, there is exactly one $\lambda>0$ for which the solution $\phi=h$ to problem $D_{1}(a, \alpha, \lambda)$ has $h(f(a))=$ $g(\alpha)$.

Proof. Suppose this fails, and there are $\lambda_{1}<\lambda_{2}$ such that the solutions $\phi_{i}$ to problems $D_{1}\left(a, \alpha, \lambda_{i}\right)(i=1,2)$ both have $\phi_{i}(f(a))=g\left(\phi_{i}(a)\right)$.

Then by Lemma 6.9 both solutions have $\phi_{i}(f(x))=g\left(\phi_{i}(x)\right)$ on $J$, both map $J$ onto $J$, and both derivatives extend continuously to 0 .

Since, initially, $\phi_{1}(a)=\phi_{2}(a)$ and $\phi_{1}^{\prime}(a)<\phi_{2}^{\prime}(a)$, we have $\phi_{1}(x)>\phi_{2}(x)$ for some distance to the left of $a$. Since $\phi_{1}(0)=\phi_{2}(0)(=0)$, there exists a first point $e<a$ at which $\phi_{1}(e)=\phi_{2}(e)$. Just to the right of $e$, we have $\phi_{1}(x)>\phi_{2}(x)$, and hence $\phi_{1}^{\prime}(e) \geq \phi_{2}^{\prime}(e)$. But this contradicts the differential equation, because (since $\phi_{1}(e)=\phi_{2}(e)$ ) we have

$$
\phi_{1}^{\prime}(e)=\lambda_{1} H\left(e, \phi_{1}(e)\right)<\lambda_{2} H\left(e, \phi_{2}(e)\right)=\phi_{2}^{\prime}(e) .
$$

This contradiction establishes the result. 
At this stage, we have completed the proof of Theorem 2.5.

Corollary 6.12. Suppose Condition (P) holds. Then there is precisely a one-parameter family of $C^{1}$ conjugations from $f$ to $g$ on $[0,+\infty)$.

Proof. In fact, if we fix $a$, there is precisely one conjugation $\phi=\Phi_{+}(a, \alpha)$ for each $\alpha \in(0,+\infty)$.

Thus there is at most a one-parameter family of $C^{\infty}$ conjugations from $f$ to $g$. (One could recover Kopell's Lemma (cf. Subsection 6.3) from this. However, it can be proved directly without all this apparatus (cf. [20, 4.1.1]). One should also remark that the corollary may be obtained directly from Kopell's Lemma, and holds for $C^{2}$ conjugations.)

6.2. Remarks about $\phi^{\prime}(0)$. Assume Condition (P) holds.

If 0 is a hyperbolic point for $f$, then the family of conjugating maps is parametrized by the multiplier at 0 . This is so, because $f$ is conjugate to $\lambda$. for $\lambda=f^{\prime}(0)$, which has centralizer consisting of all the maps $\mu \cdot$, with $\mu \neq 0$. If two maps $h$ and $k$ that conjugate $\lambda$. to $g$ have the same multiplier, then $k^{-1} h$ commutes with $\lambda$. and has multiplier 1 , and hence $h^{\prime}(0)=k^{\prime}(0)$.

If $f^{\prime}(0)=1$, but $f-x$ is not flat at 0 , then it follows from Lubin's theorem about the centralizer of a formal power series [6, 22, that the centralizer of $f$ in $\operatorname{Diffeo}([0,+\infty))$ consists of maps that have derivative 1 at 0 , and for general $g$ the family of conjugating maps from $f$ to $g$ is a coset of this centralizer. Thus all the diffeomorphic conjugations of $f$ to $g$ have the same derivative at 0 .

This does not tell us anything about merely $C^{1}$ conjugations, nor about what happens when $f-x$ is flat at 0 , but it is possible to see that again the conjugating $C^{1}$ maps all have the same derivative at 0 . The essential point is the following, which can be proved more simply now than Lemma 5.8 .

Proposition 6.13. Suppose $f \in S_{-}, f^{\prime}(0)=1$, and $\phi$ is a $C^{1}$ diffeomorphism of $[0,+\infty)$, commuting with $f$. Then $\phi^{\prime}(0)=1$.

Proof. Fix $a>0$, and let $\alpha=\phi(a)$. Then $\phi$ is $\Phi_{+}(a, \alpha)$. Let $a_{k}=f^{\circ k}(a)$ whenever $k \in \mathbb{Z}$. There is a unique $k$ such that

$$
a_{k+1} \leq \alpha<a_{k} .
$$

So at $a, \phi$ lies between $f^{\circ k}$ and $f^{\circ(k+1)}$.

If $\phi(a)=f^{\circ k}(a)$, then by Lemma 6.11 $\phi$ coincides with $f^{\circ k}$ on $J$, and hence has derivative 1 at 0 , and we are done.

Otherwise, Lemma 6.11 tells us that $\phi$ never has the same value as $f^{\circ k}$ or $f^{\circ(k+1)}$ at any point, so its graph lies sandwiched between their graphs.

Thus, since $f(0)=\phi(0)=0$,

$$
f^{\circ k}(x)>\phi(x)-\phi(0)>f^{\circ(k+1)}(x)-f^{\circ(k+1)}(0)
$$

for all $x>0$, and hence, dividing by $x$ and taking limits we get $\phi^{\prime}(0)=1$.

We remark that this result becomes trivial for $C^{2}$ conjugations. If we assume that the conjugating map is $C^{\infty}$ to 0 , then Corollary 5.3 provides a much easier way to a stronger conclusion.

Proposition 6.14 (Kopell). If $\phi \in \operatorname{Diffeo}([0,+\infty))$ commutes with $f$, and $f$ is flat at 0 , then so is $\phi$.

Proof. From Corollary 5.3, $\phi(x)-x$ tends to zero more rapidly than any power of $x$, and hence given that $\phi(x)-x$ is smooth, all its derivatives vanish at 0 . 
6.3. Remark about centralizers. The special case $f=g$ of the foregoing corresponds to results of Kopell [10 pp. 167-171] about centralizers. Indeed, Kopell made use of the $f=g$ version of the differential equation of problem $D_{1}$ in order to obtain her results. See also [13, Section 8.6, pp. 353-355]. (We have not seen the differential equation for general $f$ and $g$ used in the literature.)

The elements of the centralizer $C_{f}$ of $f$ in $\operatorname{Diffeo}([0,+\infty)$ ) (where $f$ fixes only 0 ) are exactly the elements that conjugate $f$ to $f$, so applying the foregoing to the case $g=f$, we have Kopell's result that the centralizer is at most a one-parameter group. The centralizer is never trivial, since it has all iterates $f^{\circ n}(n \in \mathbb{Z})$ as elements. However, it may fail to be connected. Sergeraert [27] gave an example in which $f$ has no smooth compositional square root, and hence its centralizer is discrete.

Sergeraert also gave a useful sufficient condition for the centralizer of an element $f \in S_{-}$to be connected. His condition is the existence of constants $\kappa>0$ and $\delta>0$ such that

$$
\sup _{0 \leq y \leq x}(y-f(y)) \leq \kappa(x-f(x)),
$$

whenever $0<x<\delta$. In particular, it always works if $x-f(x)$ is monotone.

The homomorphism $h \mapsto h^{\prime}(0)$ maps the centralizer of a given $f$ to a multiplicative subgroup of $(0,+\infty)$, but (as we've seen) the subgroup in question is just $\{1\}$, as soon as $f^{\prime}(0)=1$.

In a rather similar way, the homomorphism $\Pi: h \mapsto T_{0} h$ maps $C_{f}$ onto a subgroup of the group of invertible formal power series, and the image must have $T_{0} f$ as an element.

We have seen in Proposition 6.14 that if $f-x$ is flat at 0 , then all elements of its centralizer have the same property, so $\Pi$ is trivial.

Generally, the image of $C_{f}$ under $\Pi$ is a subgroup of the centralizer of $T_{0} f$ in the power series group. In case $T_{0} f=X \bmod X^{p+1}$ but $T_{0} f \neq X \bmod X^{p+2}$, it is a purely algebraic fact (cf. [10, p. 170]; 6] or [13, pp. 355ff]) that the latter centralizer is a oneparameter group, and indeed the map to the coefficient of $X^{p+1}$ is an isomorphism to $(\mathbb{R},+)$.

It is interesting to note in passing that the differential equation provides a way to construct smooth compositional $k$ th roots of a diffeomorphism $f \in S^{-}$of $[0,+\infty)$ that has a connected centralizer: One takes $f=g$, fixes $a>0$, and considers the initialvalue problem $D_{1}\left(a, \alpha, \Lambda_{+}(a, \alpha)\right)$ for $\alpha$ between $a$ and $f(a)$. The solution $\phi_{\alpha}$ that has $\phi^{\circ k}(a)=f(a)$ is the desired root. Since $\phi^{\circ k}(a)$ moves continuously and monotonically away from $a$ as $\alpha$ moves towards $f(a)$ from $a$, and passes $f(a)$ before $\alpha$ reaches $f(a)$, there must exist a unique $\alpha$ with the above property.

In general, if $f$ does not have roots in the diffeomorphism group, this will construct $C^{1}$ roots.

6.4. Sufficiency of $(P)$ and $(T)$ : Counterexample. The Conditions $(P)$ and $(T)$ together are not sufficient for $C^{\infty}$ conjugacy, and the following example will demonstrate this.

We have noted that in the nonflat case the existence of a $C^{1}$ conjugacy is strictly weaker than the existence of a $C^{\infty}$ conjugacy. The example will show that it is also weaker in the flat case.

Example 6.1. Consider the diffeomorphisms of $[0,+\infty)$ defined on the interior by

$$
\begin{aligned}
f(x) & =x+e^{-1 / x^{2}}, \\
\phi(x) & =x+x^{3 / 2}, \\
g & =f^{\phi} .
\end{aligned}
$$


One finds that $\phi$ is only $C^{1}$ on $[0,+\infty)$, but that $f$ and $g$ are smooth: In fact, letting $\psi=\phi^{\circ-1}$, we calculate

$$
\begin{aligned}
\psi^{\prime}(\phi) \phi^{\prime} & =1, \\
\psi^{\prime}(\phi) \phi^{\prime \prime}+\psi^{\prime \prime}(\phi)\left(\phi^{\prime}\right)^{2} & =0 .
\end{aligned}
$$

Thus

$$
\begin{aligned}
g^{\prime} & =\psi^{\prime}(f \circ \phi) f^{\prime}(\phi) \phi^{\prime}, \\
g^{\prime \prime} & =\psi^{\prime}(f \circ \phi) f^{\prime}(\phi) \phi^{\prime \prime}+\psi^{\prime}(f \circ \phi) f^{\prime \prime}(\phi)\left(\phi^{\prime}\right)^{2}+\psi^{\prime \prime}(f \circ \phi)\left\{f^{\prime}(\phi) \phi^{\prime}\right\}^{2} .
\end{aligned}
$$

The second term in the expression for $g^{\prime \prime}$ is continuous, and the other two add to

$$
f^{\prime}(\phi)\left\{\psi^{\prime}(f \circ \phi) \phi^{\prime \prime}+\psi^{\prime \prime}(f \circ \phi) f^{\prime}(\phi)\left(\phi^{\prime}\right)^{2}\right\} .
$$

The only problem is to see continuity at 0 , and the point is that for small positive $x$ we have $\phi^{\prime}(x) \approx 1, \psi^{\prime}(x) \approx 1$,

$$
\phi^{(k)}(x)=O\left(x^{\frac{3}{2}-k}\right), \quad \forall k \geq 2,
$$

and for some sequence of integers $p_{k}$,

$$
\psi^{(k)}(x)=O\left(x^{-p_{k}}\right), \quad \forall k \geq 2
$$

(as is verified inductively by differentiating (10)). Thus, since $f(x)-x$ is flat at 0 , $f^{\prime}(\phi(x))$ may be replaced by 1 and $f \circ \phi$ by $\phi$, in the expression (111), with an error that is $O\left(x^{N}\right)$ for all $N \in \mathbb{N}$. But when this is done we just get 0 , by (10), so $g^{\prime \prime} \rightarrow 0$ as $x \rightarrow 0$.

It now becomes clear that when we continue to differentiate $g$, and express $g^{(k)}$ in terms of $\psi, f$, and $\phi$, we get an expression involving derivatives of $\psi$ (at $f \circ \phi$ ), $f$ (at $\phi$ ), and $\phi$, and that when $f$ is replaced by $\mathbb{1}$ in this expression we get zero (the $k$ th derivative of $\psi \circ \phi)$. Moreover, for small $x$, the error involved in replacing $f(\phi)$ by $\phi, f^{\prime}(\phi)$ by 1 , $f^{\prime \prime}(\phi)$ by 0 , and all higher derivatives $f^{(k)}(\phi)$ by 0 , is $O\left(x^{N}\right)$ for all $N$. Thus $g^{(k)} \rightarrow 0$ as $x \rightarrow 0$ for all $k \geq 3$, as well. It follows that $g$ is $C^{\infty}$, and $g(x)-x$ is flat at 0 , as required.

Now any other $C^{1}$ conjugation of $f$ and $g$ will differ from $\phi$ by composition with an element of the centralizer of $f$. Since $f(x)-x$ is monotone, it satisfies Sergeraert's condition 27. p. 259, Theorem 3.1], and hence the centralizer of $f$ consists of $C^{\infty}$ diffeomorphisms, and hence no conjugation of $f$ to $g$ is better than $C^{1}$.

This shows that Conditions $(\mathrm{P})$ and $(\mathrm{T})$ are not sufficient, by themselves, to guarantee conjugacy, in general.

Question. Since not all $C^{1}$ conjugacies between given $f$ and $g$ in $\operatorname{Diffeo}([0, \infty))$ are $C^{\infty}$ to zero, it would be interesting to know whether or not the set of parameters $\alpha$ for which the solution $\Phi_{+}(a, \alpha)$ is $C^{\infty}$ to zero is always a relatively closed subset of $(0, \infty)$. We were not able to resolve this question 3

\section{§7. Compact intervals}

Recall from Subsection 2.8 that in the context of a compact interval the meaning of Condition $(\mathrm{P})$ must now be modified and that it now involves a two-sided product.

\footnotetext{
${ }^{3}$ The question has now been answered by H. Eynard [35], who constructed an element $f \in$ $\operatorname{Diffeo}([0, \infty))$ whose $C^{2}$ centralizer is an uncounted proper subset of its $C^{1}$ centralizer. Taking $g=f$, we see that this implies that the set of parameters $\alpha$ for which $\Phi_{+}(a, \alpha)$ is $C^{\infty}$ to zero is not relatively closed in $(0, \infty)$.
} 
7.1. The sign condition and Condition $(\mathbf{P})$. Let $I=[d, c]$ be a compact interval with interior $J$. Let $f$ and $g$ belong to $\operatorname{Diffeo}^{+}(I)$. The sign condition is, as before, necessary for conjugacy of $f$ and $g$.

Applying Lemma 5.2 and Corollary 5.4 to the inverse maps $f^{\circ-1}$ and $g^{\circ-1}$ on the half-open interval $J \cup\{c\}$, we see that similar results hold for the products

$$
\prod_{n=1}^{\infty} \frac{f^{\prime}\left(x_{-n}\right)}{f^{\prime}\left(y_{-n}\right)} \text { and } \prod_{n=1}^{\infty} \frac{f^{\prime}\left(f^{\circ-n}(x)\right)}{g^{\prime}\left(g^{\circ-n}(\xi)\right)} .
$$

Thus we obtain:

Lemma 7.1. Suppose $I$ is compact and $f$ and $g$ are conjugate in $\operatorname{Diffeo}^{+}(I)$. Then

$$
\prod_{n=0}^{\infty} \frac{f^{\prime}\left(f^{\circ n}(x)\right)}{g^{\prime}\left(g^{\circ n}(\xi)\right)}
$$

converges for each $x, \xi \in J$, and

$$
\prod_{n=1}^{\infty} \frac{f^{\prime}\left(f^{\circ-n}(x)\right)}{g^{\prime}\left(g^{\circ-n}(\xi)\right)}
$$

converges for each $x, \xi \in J$.

The proof of Lemma 5.1 gives:

Corollary 7.2. If $f=g^{h}$, then the two-sided product

$$
\prod_{n=-\infty}^{\infty} \frac{f^{\prime}\left(f^{\circ n}(x)\right)}{g^{\prime}\left(g^{\circ n}(h(x))\right)}
$$

is independent of $x \in J$ and equals the ratio $h^{\prime}(c) / h^{\prime}(d)$ of the derivatives of the conjugating map at the ends.

7.2. The differential equation. Suppose $f, g \in \operatorname{Diffeo}^{+}(I)$ satisfy the sign condition and Condition $(\mathrm{P})$.

Definition. We define

$$
\begin{aligned}
& F_{1 a}(x)=H_{1}(f, f, x, a)=\prod_{n=0}^{\infty} \frac{f^{\prime}\left(f^{\circ n}(x)\right)}{f^{\prime}\left(f^{\circ n}(a)\right)}, \\
& G_{1 \alpha}(\xi)=H_{1}(g, g, \xi, \alpha)=\prod_{n=0}^{\infty} \frac{g^{\prime}\left(g^{\circ n}(\xi)\right)}{g^{\prime}\left(g^{\circ n}(\alpha)\right)}, \\
& F_{2 a}(x)=H_{2}(f, f, a, x)=\prod_{n=1}^{\infty} \frac{f^{\prime}\left(f^{\circ-n}(x)\right)}{f^{\prime}\left(f^{\circ-n}(a)\right)} \\
& G_{2 \alpha}(\xi)=H_{2}(g, g, \alpha, \xi)=\prod_{n=1}^{\infty} \frac{g^{\prime}\left(g^{\circ-n}(\xi)\right)}{g^{\prime}\left(g^{\circ-n}(\alpha)\right)}
\end{aligned}
$$

whenever $x, \xi, a, \alpha \in J$. 
Applying Lemma 6.1 to the original maps and to their inverses, we obtain:

Lemma 7.3. Let the sign condition and $(\mathrm{P})$ hold, and fix $a, \alpha \in J$. Then

(1) the functions $F_{1 a}, G_{1 \alpha}, F_{2 a}$, and $G_{2 \alpha}$ are infinitely differentiable and positive on $J$, and hence

(2) $H_{1}(x, \xi)=H_{1}(a, \alpha) \cdot F_{1 a}(x) / G_{1 \alpha}(\xi)$ and $H_{2}(x, \xi)=H_{2}(a, \alpha) \cdot G_{2 \alpha}(\xi) / F_{2 a}(x)$ are infinitely differentiable and positive on $J \times J$.

We now consider two three-parameter initial-value problems (2) and (4):

$$
\begin{array}{ll}
D_{1}(a, \alpha, \lambda): & \left\{\begin{array}{l}
\frac{d \phi}{d x}=H_{1}(x, \phi(x)) \lambda, \\
\phi(a)=\alpha,
\end{array}\right. \\
D_{2}(a, \alpha, \mu): & \left\{\begin{array}{l}
\frac{d \phi}{d x}=H_{2}(x, \phi(x)) \mu, \\
\phi(a)=\alpha .
\end{array}\right.
\end{array}
$$

Applying Lemma 5.2 and Corollary 5.2 twice, we obtain:

Lemma 7.4. Suppose $f, g, h \in \operatorname{Diffeo}^{+}(I)$ and $f=g^{h}$. Then

(1) the restriction $\phi=h \mid J$ is the solution to problem $D_{1}\left(a, h(a), h^{\prime}(d)\right)$, whenever $a \in J$

(2) the same $\phi$ is the solution to problem $D_{2}\left(a, h(a), h^{\prime}(c)^{-1}\right)$, whenever $a \in J$;

(3) the function $a \mapsto H(a, h(a))$ is constant on $J$, equal to $h^{\prime}(c) / h^{\prime}(d)$.

To characterize the existence of a conjugating $h$, we need to formulate the conditions of this lemma in a way that does not refer explicitly to $h$. As before, we can do this by using the differential equations. The following is a consequence of the series of lemmas from the last section.

Lemma 7.5. Assume the sign condition and Condition $(\mathrm{P})$.

(1) For each $a \in J$, and each $\alpha \in J$, there exists a unique $\lambda>0$ such that the unique solution $\phi$ to problem $D_{1}(a, \alpha, \lambda)$ has $\phi(f(a))=g(\alpha)$. This $\phi$ is a bijection of $J$ onto $J$, and has a one-sided derivative at $d$, with

$$
\lim _{x \rightarrow d} \phi^{\prime}(x)=\phi^{\prime}(d)=\lambda .
$$

(2) For each $a \in J$, and each $\alpha \in J$, there exists a unique $\mu>0$ such that the unique solution $\psi$ to problem $D_{2}(a, \alpha, \mu)$ has $\psi(f(a))=g(\alpha)$. This $\psi$ is a bijection of $J$ onto $J$, and has a one-sided derivative at $c$, with

$$
\lim _{x \rightarrow c} \psi^{\prime}(x)=\psi^{\prime}(c)=\mu .
$$

So either initial-value problem together with the conjugation equation at one point is enough to guarantee the conjugation equation on the whole interior $J$.

With the notation of the last lemma, recall that we denote the unique $\lambda$ of part (1) by $\Lambda_{+}(a, \alpha)$, and the corresponding $\phi(x)$ by $\Phi_{+}(a, \alpha ; x)$. Similarly, we denote the $\mu$ of part $(2)$ by $\Lambda_{-}(a, \alpha)$ and the $\psi$ by $\Phi_{-}(a, \alpha ; x)$.

7.3. Extending a conjugation to ends of $I$. Assuming the sign condition and $(\mathrm{P})$, we consider the following condition.

Condition (E). There exist $a, \alpha \in J$, for which the solution $\phi=\Phi_{+}(a, \alpha ; \cdot)$ has a $C^{\infty}$ extension to $I$ (and hence agrees with the solution $\Phi_{-}(a, \alpha ; \cdot)$ ).

It is clearly equivalent to replace "there exist $a, \alpha$ " by "for each $a$ there exists $\alpha$ " in the formulation of Condition (E). 
We note the following:

Lemma 7.6. Suppose the sign Condition, $(\mathrm{P})$, and $(\mathrm{E})$. Then if $h=\phi$ is a solution to problem $D_{1}(a, \alpha, \lambda)$ with $g(\alpha)=\phi(f(a))$ and extends smoothly to the ends of $J$, it follows that

(1) $h^{\prime}(d)=\lambda$;

(2) $f=g^{h}$ on $\operatorname{clos} J$

(3) $h$ is a solution to problem $D_{2}\left(a, \alpha, h^{\prime}(c)\right)$;

(4) $h^{\prime}(c)=H(a, \alpha) h^{\prime}(d)$.

The proof of Theorem 2.8 is now complete.

7.4. Functional moduli. Robbin [25, p. 424] described the solution to the conjugacy problem on a compact interval, subject to the sign condition, in the case when both ends are hyperbolic fixed points, i.e., when $f^{\prime}(c) \neq 1 \neq f^{\prime}(d)$. (See also [3] and 12, Chapter 2].)

In that case, Condition (P) reduces to the two equations $f^{\prime}(c)=g^{\prime}(c)$ and $f^{\prime}(d)=$ $g^{\prime}(d)$, and conjugacy may be characterized in terms of a modulus. Robbin's modulus is a diffeomorphism of $(0,+\infty)$. He constructs the modulus for $f$ by linearizing the restrictions of $f$ to $\{c\} \cup J$ and to $J \cup\{d\}$, i.e., choosing the (unique) $\alpha_{f}:\{c\} \cup J \rightarrow$ $[0,+\infty)$ and $\beta_{f}: J \cup\{d\} \rightarrow[0,+\infty)$ such that

$$
\begin{aligned}
& \alpha(f(x))=f^{\prime}(c) \cdot \alpha(x), \\
& \beta(f(x))=f^{\prime}(d) \cdot \beta(x),
\end{aligned}
$$

whenever $x \in J$, and $\alpha^{\prime}(c)=\beta^{\prime}(d)=1$. His modulus is $\gamma_{f}=\beta \circ \alpha^{\circ-1}$. The two maps $f$ and $g$ are conjugate if and only if $\gamma_{f}=\gamma_{g}$. Thus Robbin's modulus serves to label the elements of the uncountable family of conjugacy classes with respect to the group Diffeo $^{+}(I)$ on the compact interval into which each single conjugacy class with respect to the group Diffeo ${ }^{+}(J \cup\{c\})$ on one of the half-open intervals splits.

One could try to construct an invariant composed of Taylor series conjugacy classes and a modulus, for the general nonflat cases. In fact, Young 34 has shown that the conventional multiplier introduced by Afraimovitch, Liu and Young 1 can be used to make a modulus for the "saddle-node" case (in which $f(x)-f(p)$ vanishes to precisely second order at the ends $p$ of $I$. We expect that this works as soon as $f-x$ is not flat at either end.

Afraimovitch et al. associated with a suitable diffeomorphism $f \in S$ and any fixed $a \in J$ the functions

$$
\begin{aligned}
& u_{+}(x)=\lim _{n \uparrow+\infty} \frac{f^{\circ n}(x)-f^{\circ n}(a)}{f^{\circ(n+1)}(a)-f^{\circ n}(a)}, \\
& u_{-}(x)=\lim _{n \downarrow-\infty} \frac{f^{\circ n}(x)-f^{\circ n}(a)}{f^{\circ(n+1)}(a)-f^{\circ n}(a)},
\end{aligned}
$$

defined for each $x \in J$. They showed that the limits exist when $f-x$ is not flat at 0 . Also, in that case, each of $u_{ \pm}$is a smooth bijection of $J$ onto $\mathbb{R}$, and one thinks of $u_{ \pm}$as "new coordinates" on $J$, adapted to $f$.

It is possible to continue this process, to develop moduli for more of Takens' cases, and for diffeomorphisms that are flat at the ends. However the flat (and semi-flat) cases offer enormous variety, and Condition (E) as it stands seems the simplest way to express the obstruction to smooth conjugacy, given $C^{1}$ conjugacy. The modulus conditions are computable in principle, but the computations are massive. 


\section{§8. Conjugacy in Diffeo ${ }_{B}^{+}$}

Throughout this section, $I$ will be one of $\mathbb{R},[-1,+\infty)$, or $[-1,1]$. (Each interval with nonempty interior is diffeomorphic to one of these, and it will be convenient to have 0 in the interior of $I$.)

$E$ will be a fixed closed nonempty subset of $I$, containing any ends that $I$ has, and $B$ will be the boundary of $E$.

8.1. Proof of Lemma 2.15. The end $p$ in question is an accumulation point of $E$, and hence $f-x$ and $g-x$ are flat there. By assumption, there exists some $\phi_{0} \in$ Diffeo $_{B}^{+}$that conjugates $g$ to $f$ on $I$. Since $\phi_{0}$ fixes each point of $B, \phi_{0}-x$ is flat at $p$. If $\phi$ is another map that conjugates $f$ to $g$ on $I$, then $\phi_{0}^{\circ-1} \circ \phi$ belongs to the centralizer $C_{f}$ of $f$ in Diffeo $_{B}^{+}$. Since $f-x$ is flat at $p$, so is every element of $C_{f}$ by Proposition 6.14 Thus $\phi$ is the composition of two functions that fix $p$ and have Taylor series $X$ there, and the result follows.

8.2. Proof of Theorem 2.16, We need only prove the "if" part.

Assume Conditions (M) and (F).

We define a variation of $\operatorname{Conj}(f, g, J)$, corresponding to conjugations that fix each point of $B$.

Definition. If $L$ is a connected component of $I \sim B^{\prime}$, we denote the set of all maps that conjugate $f$ to $g$ in $\operatorname{Diffeo}_{B}^{+}(\operatorname{clos}(L))$ by $\operatorname{Conj}_{B}(f, g ; L)$.

The assumption (M) tells us that each $\operatorname{Conj}_{B}(f, g ; L)$ is nonempty. We have to show that we can patch together elements of the various $\operatorname{Conj}_{B}(f, g ; L)$ to get an element of $\operatorname{Diffeo}^{+}(I)$.

We claim that for each $L$, we may choose $h \in \operatorname{Conj}_{B}(f, g ; L)$ with $h-x$ flat at each end of $L$ that is not an end of $I$.

Let $p$ be an end of $L$ that is not an end of $I$. Then $p \in B^{\prime}$. There are three cases.

1. $p$ is an accumulation point of $L \cap B$. Then since all elements of $\operatorname{Conj}_{B}(f, g ; L)$ fix all points of $L \cap B$, they all have $h-x$ flat at $p$.

2. $p$ is isolated in $L \cap B$, and $p$ is an end of some component $J \subset L$ of $I \sim E$ (note that $p$ must be a limit of points of $B$ that lie on the other side of $p$ from $J$ ). Then Condition (F) tells us that all elements of $\operatorname{Conj}(f, g ; J)$ have $h-x$ flat at $p$. Thus all elements of $\operatorname{Conj}(f, g ; L)$ also have $h-x$ flat at $p$.

3. $p$ is isolated in $L \cap B$, and $p$ is an end of some component $J \subset L$ of the interior of $E$. Then given any $h \in \operatorname{Conj}(f, g ; L)$, we may modify it on $J$ in any way at all (provided it remains a diffeomorphism of $J$ onto itself) without disturbing the conjugacy, because $f(x)=g(x)=x$ on $J$. Thus we can modify it to make $h-x$ flat at $p$.

So the claim holds. So if we choose $h$ on each $L$ to have $h-x$ flat at each end in the interior of $I$, then they automatically fit together to make the desired conjugation.

\section{§9. Reducing from Diffeo $(I)$ to $\operatorname{Diffeo}^{+}(I)$}

In this section we discuss the reduction of the conjugacy problem in the full diffeomorphism group to the conjugacy problem in the subgroup of direction-preserving maps.

There is no issue for half-open intervals, since the two groups coincide, so it suffices to consider the two cases $I=\mathbb{R}$ and $I=[-1,1]$, which represent all other intervals up to diffeomorphism. (It is convenient to use representatives that are invariant under -.)

9.1. Reducing to conjugation by elements of $\mathrm{Diffeo}^{+}$. The first (simple) proposition allows us to restrict attention to conjugation using $h \in \operatorname{Diffeo}^{+}(I)$. 
Proposition 9.1. Let $I=\mathbb{R}$ or $[-1,1]$. Let $f, g \in \operatorname{Diffeo}(I)$. Then the following two conditions are equivalent:

(1) There exists $h \in \operatorname{Diffeo}(I)$ such that $f=g^{h}$.

(2) There exists $h \in \operatorname{Diffeo}^{+}(I)$ such that $f=g^{h}$ or $-\circ f \circ-=g^{h}$.

Proof. If (1) holds, and $\operatorname{deg} h=-1$, then $-\circ f \circ-=g^{k}$, with

$$
k(x)=h(-x) .
$$

The rest is obvious.

9.2. Reducing to conjugation of elements of $\mathrm{Diffeo}^{+}$. The degree of a diffeomorphism is a conjugacy invariant, so to complete the reduction of the conjugacy problem in Diffeo to the problem in Diffeo ${ }^{+}$, it suffices to deal with the case when $\operatorname{deg} f=\operatorname{deg} g=-1$ and $\operatorname{deg} h=+1$.

Note that fix $(f)$ and fix $(g)$ are singletons and lie in $\operatorname{int}(I)$. If $f=g^{h}$, then $h(\operatorname{fix}(f))=$ fix $(g)$, and (since Diffeo ${ }^{+}$acts transitively on $\operatorname{int}(I)$ ) we may thus, without loss in generality, suppose that $f(0)=g(0)=h(0)=0$.

If $f=g^{h}$, then we also have $f^{\circ 2}=\left(g^{\circ 2}\right)^{h}, f^{-1}=\left(g^{-1}\right)^{h}$, and $f^{\circ 2} \in$ Diffeo $^{+}$.

We have the following reduction.

Theorem 9.2. Let $I=\mathbb{R}$ or $[-1,1]$. Suppose $f, g \in$ Diffeo $^{-}$, fixing 0 . Then the following two conditions are equivalent:

1. $f=g^{h}$ for some $h \in$ Diffeo $^{+}$.

2. (a) There exists $h_{1} \in$ Diffeo $_{0}^{+}$such that $f^{\circ 2}=\left(g^{\circ 2}\right)^{h_{1}}$ and

(b) letting $g_{1}=g^{h_{1}}$, there exists $h_{2} \in$ Diffeo $^{+}$, commuting with $f^{\circ 2}$ and fixing 0 , such that $T_{0} f=\left(T_{0} g_{1}\right)^{T_{0} h_{2}}$.

See [23, Proposition 2.1] for details.

9.3. Making the conditions explicit. To complete the project of reducing conjugation in Diffeo to conjugation in Diffeo $^{+}$, we have to find an effective way to check condition 2(b). In other words, we have to replace the nonconstructive "there exists $h_{2} \in$ Diffeo $^{+}$" by some condition that can be checked algorithmically. This is achieved by the following:

Theorem 9.3. Let $I=\mathbb{R}$ or $[-1,1]$. Suppose that $f, g \in \mathrm{Diffeo}^{-}$both fix 0 , and have $f^{\circ 2}=g^{\circ 2}$. Then there exists $h \in$ Diffeo $^{+}$, commuting with $f^{\circ 2}$, such that $T_{0} f=\left(T_{0} g\right)^{T_{0}} h$ if and only if one of the following holds:

(1) $\left(T_{0} f\right)^{\circ 2} \neq X$;

(2) 0 is an interior point of $\operatorname{fix}\left(f^{\circ 2}\right)$;

(3) $\left(T_{0} f\right)^{\circ 2}=X, 0$ is a boundary point of fix $\left(f^{\circ 2}\right)$, and $T_{0} f=T_{0} g$.

Note that the conditions (1)-(3) are mutually exclusive. We record a couple of corollaries.

Corollary 9.4. Suppose that $f, g \in$ Diffeo $^{-}$fix 0 , and that $\left(T_{0} f\right)^{\circ 2} \neq X$ or $0 \in$ $\operatorname{int}(f i x(f))$. Then $f=g^{h}$ for some $h \in \mathrm{Diffeo}^{+}$if and only if $f^{\circ 2}=\left(g^{\circ 2}\right)^{h}$ for some $h \in$ Diffeo $^{+}$.

In case $\left(T_{0} f\right)^{\circ 2} \neq X$, any $h$ that conjugates $f^{\circ 2}$ to $g^{\circ 2}$ will also conjugate $f$ to $g$. In the other case covered by this corollary, it is usually necessary to modify $h$ near 0 .

Corollary 9.5. Suppose that $f, g \in$ Diffeo $^{-}$fix 0 , and that $\left(T_{0} f\right)^{\circ 2}=X$ and $0 \in$ $\operatorname{bdy}(\operatorname{fix}(f))$. Then $f=g^{h}$ for some $h \in \mathrm{Diffeo}^{+}$if and only if $f^{\circ 2}=\left(g^{\circ 2}\right)^{h}$ for some $h \in$ Diffeo $^{+}$and $T_{0} f=T_{0} g$. 
The last corollary covers the case where 0 is isolated in $\operatorname{fix}\left(f^{\circ 2}\right)$ and $T_{0} f$ is involutive, as well as the case where 0 is both an accumulation point and a boundary point of fix $(f)$.

The detailed proofs may be found in [23. They use results about conjugacy and reversibility for formal power series, together with Kopell's results about centralizers.

\section{§10. Further EXamples AND REMARKS}

10.1. Reduction to fix $(f)=\operatorname{fix}(g)$. In relation to the reduction of Subsection 2.12, it is not true that each map $h$ conjugating $g$ to $f$ may be factored as any smooth map that maps fix $(f)$ onto fix $(g)$, followed by a smooth map fixing fix $(f)$.

Example 10.1. Take, for instance $f(x)=x+\sin (x) / 10$, and $g(x)=x-\sin (x) / 10$. Both fix precisely $\pi \mathbb{Z}$. They are conjugated by $h: x \mapsto x-\pi$. The identity map $h_{1}$ maps fix $(f)$ onto fix $(g)$, but no map fixing fix $(f)$ conjugates $f$ to $g$, since the multipliers are wrong.

In general, in searching for a factor $h_{1}$ as in Proposition 2.13. we may start by classifying the points $p$ of $\operatorname{bdy}(f i x(f))$ (and bdy $(f i x(g))$ ) according to the conjugacy class of $T_{p} f$ (or $T_{p}(g)$ ). This produces two classifications, the $f$-classification of fix $(f)$, and the $g$-classification of fix $(g)$. Only maps $h_{1}$ that respect these classifications are eligible as potential factors. Precisely speaking, the eligible maps $h_{1}$ must be such that $T_{p} f$ and $T_{h_{1}(p)} g$ are conjugate Taylor series, for each $p \in \operatorname{bdy}(\operatorname{fix}(f))$.

Example 10.2. For instance, if we modified the above example by taking

$$
f(x)=x+\frac{\sin x}{1+x^{4}}
$$

then there is no eligible map at all, so $f$ and $g$ are not conjugate.

Example 10.3. If we modified $g$ as well, taking

$$
g(x)=x+\frac{\sin 2 x}{2+8 x^{2}} \quad\left(=\frac{1}{2} f(2 x)\right),
$$

then the only eligible $h_{1}$ are those that have $h_{1}(x)=2 x$ on $\operatorname{fix}(f)=\pi \mathbb{Z}$.

This prompts the question, whether, assuming the maps $f$ and $g$ are conjugate, $e v$ ery $h_{1} \in$ Diffeo $^{+}$that respects this Taylor-series classification at the boundary points will serve as a factor of the kind referred to in Proposition 2.13. That would be very convenient, as it would characterize the diffeomorphisms $h_{1}$ that we need to find. Unfortunately, the answer is no.

Example 10.4. Take any $f \in \operatorname{Diffeo}^{+}(\mathbb{R})$ that fixes precisely $\mathbb{Z}$, has $f-x$ flat at each integer, and is such that the functions on $[0,1]$ defined by $x \mapsto f(x+n)-n(n \in \mathbb{Z})$ represent distinct conjugacy classes of $\operatorname{Diffeo}^{+}([0,1])$. Take $g(x)=1+f(x-1)$. Then $f$ and $g$ are conjugate, but the map $h_{1}(x)=x+2$ won't do as a factor of the required kind, because no map that fixes $\mathbb{Z}$ will conjugate $g$ to $x \mapsto 2+f(x-2)$.

It is not essential to use a function $f$ that is flat on the boundary to give an example of this kind. We know that in the nonflat case, Condition (T) is not enough to characterize conjugacy in $\operatorname{Diffeo}^{+}(I)$, for compact $I$, so we can modify the example to produce the same end result without having $f-x$ flat at all. The point is that once we have a $C^{\infty}$ diffeomorphism on each interval $[n, n+1]$ and the two available Taylor series agree at each $n$, then they patch together to make a global diffeomorphism.

So there is a substantial problem, from the constructive point of view, concerning how to search for suitable $h_{1}$.

However, we know, from Subsection 7.4 that part (3) of Proposition 2.13 can only work if $f_{1}$ and $g$ have the same $J$-modulus, for each component $J$. This provides a fine 
filter, to cut down the search, because given the Taylor series at one end, there is at most a one-parameter coset of diffeomorphisms of $J$ that conjugate $f_{1}$ to $g$ on $J$. Generically, the coset is discrete.

10.2. Finite fix $(f)$. We conclude with a summary of our conclusions about the conjugacy problem in the special case when $\operatorname{fix}(f)=\operatorname{fix}(g)=E=B$ is a finite set of points $p_{1}<p_{2}<\cdots<p_{k}$, so that there is only one way to map $E=B$ to itself, preserving order.

The case when all the points are hyperbolic is classical and has been discussed previously by Belitskir [3. We include this case in the discussion, for completeness. The first necessary condition is that Condition $(\mathrm{T})$ holds at each of the fixed points, i.e., that the Taylor series of $f$ and $g$ be conjugate. At hyperbolic points for $f$ or $g$, this amounts to the identity of the multipliers, and at the remaining points $p$ at which $f-x$ is not flat ("Takens' points"), it is determined by examining the coefficients of $f$ and $g$ up as far as the term in $X^{2 p+1}$, where $T_{p} f-X$ vanishes to order $p$, but not to order $p+1$. At the points where $f-x$ is flat, the condition is automatic.

Next, we need the sign condition that the graphs of $f$ and $g$ lie on the same side of the diagonal on each interval complementary to the fixed-point set. This condition follows automatically from $(\mathrm{T})$ at the hyperbolic and Takens' points.

Next, we need Condition (P), the convergence of the products (1). This is automatic at the hyperbolic and Takens' points, but imposes restrictions to the right and left of the points where $f-x$ is flat.

Next, we need Condition (E), to the effect that the $C^{1}$ conjugacies that now exist include some that are $C^{\infty}$ when restricted to each of the half-open intervals $\left(-\infty, p_{1}\right]$, $\left[p_{1}, p_{2}\right),\left(p_{1}, p_{2}\right],\left[p_{2}, p_{3}\right),\left(p_{2}, p_{3}\right], \ldots,\left[p_{k},+\infty\right) 4$ and that on each of the compact intervals $\left[p_{1}, p_{2}\right], \ldots,\left[p_{k-1}, p_{k}\right]$ there is at least one of these conjugacies that is smooth to both ends. Note that this means that the maps $f$ and $g$ share the same Robbin invariant.

At this stage, we have nonempty cosets $\operatorname{Conj}(J)=\operatorname{Conj}(f, g ; J)$ of maps that conjugate smoothly on each clos $J$. Next we need Condition (M), that we can match some Taylor series from $\operatorname{Conj}(J)$ and $\operatorname{Conj}\left(J^{\prime}\right)$ whenever $J$ and $J^{\prime}$ are adjacent components. This may still not be enough to make $f$ and $g$ conjugate.

We distinguish the hyperbolic and Takens' points from the points where $f-x$ is flat. At the latter, all smooth $J$-conjugations share the same Taylor series, as do all $J^{\prime}$-conjugations, so if any series from $\operatorname{Conj}(J)$ coincides with a series from $\operatorname{Conj}\left(J^{\prime}\right)$, then all do, so we can stop worrying about these fixed points.

Let $q_{1}<\cdots<q_{r}$ be the remaining fixed points, the ones at which $f-x$ is not flat. Write $L_{i}=\left(-\infty, q_{i}\right], J_{i}=\left[q_{i}, q_{i+1}\right](i<r-1)$ and $J_{r}=\left[q_{r},+\infty\right)$.

If $r=1$, we are done; $f$ and $g$ are smoothly conjugate. Otherwise $\operatorname{Conj}\left(L_{2}\right)$ is already nonempty. We have to assume that $\operatorname{Conj}\left(L_{2}\right) \cap \operatorname{Conj}\left(J_{2}\right)=\operatorname{Conj}\left(L_{3}\right)$ is nonempty; otherwise $f$ and $g$ are not conjugate. Checking this condition is a matter of comparing the set of multipliers (at hyperbolic points) or the set of $(2 p+1)$-st order Taylor polynomials (at Takens' points). Each of these sets is a coset of a group. In the hyperbolic case, unless one of the sets is the full multiplicative group $(0,+\infty)$, we are comparing two sets of the form

$$
\left\{\alpha \lambda^{n}: n \in \mathbb{Z}\right\} \text { and }\left\{\beta \mu^{n}: n \in \mathbb{Z}\right\}
$$

(i.e., two cosets of discrete subgroups of $(0,+\infty))$. In the Takens case, once we conjugate the series to canonical form, we are comparing the coefficients of $X^{p+1}$, which are two cosets of the additive group $\mathbb{R}$.

\footnotetext{
${ }^{4}$ This much is automatic at the hyperbolic and Takens' points.
} 
Continuing, we get a decreasing sequence $\operatorname{Conj}\left(L_{4}\right), \ldots, \operatorname{Conj}\left(L_{r}\right)$, and if at any stage it is empty, there is no conjugacy.

If $\operatorname{Conj}\left(L_{r}\right)$ is nonempty, the last step is to see whether $\operatorname{Conj}\left(L_{r}\right) \cap \operatorname{Conj}\left(J_{r}\right)$ is nonempty. If this last condition holds, then there is a smooth conjugacy between $f$ and $g$, and otherwise not.

\section{ACKNOWLEDGMENT}

The authors are most grateful to Étienne Ghys and Ian Short for useful advice, and to John Mather for making available his unpublished work.

\section{REFERENCES}

[1] V. Afraimovitch, W. S. Liu, and T. Young, Conventional multipliers for homoclinic orbits, Nonlinearity 9 (1996), 115-136. MR 1374001 (97d:58142)

[2] P. Ahern and J.-P. Rosay, Entire functions, in the classification of differentiable germs tangent to the identity, in one or two variables, Trans. Amer. Math. Soc. 347 (1995), 543-572. MR1276933 (95d:30038)

[3] G. R. Belitskiǔ, Smooth classification of one-dimensional diffeomorphisms with hyperbolic fixed points, Sibirsk. Mat. Zh. 27 (1986), no. 6, 21-24; English transl., Siberian Math. J. 27 (1986), 801-804. MR0883578 (88f:58117)

[4] W. E. Boyce and R. C. DiPrima, Elementary differential equations and boundary value problems, 2nd ed., Wiley, New York, 1969. MR.0179403 (31:3651)

[5] L. Carleson and T. W. Gamelin, Complex dynamics, Springer-Verlag, New York, 1993. MR1230383 (94h:30033)

[6] J. Lubin, Non-Archimedean dynamical systems, Compositio Math. 94 (1994), 321-346. MR1310863 (96g:11140)

[7] A. B. Calica, Reversible homeomorphisms of the real line, Pacific J. Math. 39 (1971), 79-87. MR0307144 (46:6265)

[8] W. de Melo and S. van Strien, One-dimensional dynamics, Ergeb. Math. Grenzgeb. (3), Bd. 25, Springer, Berlin, 1993. MR1239171 (95a:58035)

[9] D. B. A. Epstein, The simplicity of certain groups of homeomorphisms, Compositio Math. 22 (1970), 165-173. MR0267589(42:2491)

[10] N. Kopell, Commuting diffeomorphisms, Global Analysis, Proc. Sympos. Pure Math., vol. 14, Amer. Math. Soc., Providence, RI, 1970, pp. 165-184. MR.0270396 (42:5285)

[11] E. Kasner, Conformal classification of analytic arcs or elements: Poincaré's local problem of conformal geometry, Trans. Amer. Math. Soc. 16 (1915), 333-349. MR.1501016

[12] A. Katok and B. Hasselblatt, Introduction to the modern theory of dynamical systems, Encyclopedia Math. Appl., vol. 54, Cambridge Univ. Press, Cambridge, 1995. MR1326374 (96c:58055)

[13] M. Kuczma, B. Choczewski, and R. Ger, Iterative functional equations, Encyclopedia Math. Appl., vol. 32, Cambridge Univ. Press, Cambridge, 1990. MR1067720 (92f:39002)

[14] J. Mather, Commutators of diffeomorphisms, Comment. Math. Helv. 49 (1974), 512-528. MR0356129(50:8600)

[15] Commutators of $C^{r}$ diffeomorphisms of the real line (unpublished preprint, privately communicated).

[16] , On Haefliger's classifying space. I, Bull. Amer. Math. Soc. 77 (1971), 1111-1115. MR0283817 (44:1047)

[17] , Integrability in codimension 1, Comment. Math. Helv. 48 (1973), 195-233. MR0356085 (50:8556)

[18] H. Mirkil, Differentiable functions, formal power series, and moments, Proc. Amer. Math. Soc. 7 (1956), 650-652. MR0079064(18:23g)

[19] A. Navas, Groupes résolubles de difféomorphismes de l'intervalle, du cercle et de la droite, Bull. Braz. Math. Soc. (N.S.) 35 (2004), 13-50. MR2057043 (2005i:57044)

[20] _ Grupos de difeomorfismos del circulo, Ensaios Mat., vol. 13, Soc. Brazil. Mat., Rio de Janeiro, 2007. MR2394157

[21] A. G. O'Farrell, Conjugacy, involutions, and reversibility for real homeomorphisms, Irish Math. Soc. Bull. 54 (2004), 41-52. MR2138430 (2006b:37069)

[22] _ Composition of involutive power series, and reversible series, Comput. Methods Funct. Theory 8 (2008), 173-193. MR2419471 (2009e:20096) 
A. G. O'FARRELL AND M. ROGINSKAYA

[23] A. G. O'Farrell and M. Roginskaya, Reducing conjugacy in the full diffeomorphism group of $\mathbb{R}$ to conjugacy in the subgroup of orientation-preserving maps, Zap. Nauchn. Sem. S.-Peterburg. Otdel. Mat. Inst. Steklov. (POMI) 360 (2008), 231-237; English transl., J. Math. Sci. 158 (2009), 895-898.

[24] A. G. O'Farrell and I. Short, Reversibility in the diffeomorphism group of the real line, Publ. Math. 53 (2009), 401-415. MR2543857 (2010h:37041)

[25] J. W. Robbin, Unfoldings of discrete dynamical systems, Ergodic Theory Dynam. Systems 4 (1984), 421-486. MR:0776878 (87a:58117)

[26] W. Rudin, Real and complex analysis, 3rd ed., McGraw-Hill Book Co., New York, 1987. MR0924157 (88k:00002)

[27] F. Sergeraert, Feuilletages et difféomorphismes infiniment tangents à l'identité, Invent. Math. 39 (1977), 253-275. MR0474327 (57:13973)

[28] S. Sternberg, Local $C^{n}$ transformations of the real line, Duke Math. J. 24 (1957), 97-102. MR0102581(21:1371)

[29] G. Szekeres, Regular iteration of real and complex functions, Acta Math. 100 (1958), 203-258. MR0107016 (21:5744)

[30] F. Takens, Normal forms for certain singularities of vectorfields, Ann. Inst. Fourier (Grenoble) 23 (1973), 163-195. MR0365620(51:1872)

[31] S. M. Voronin, Analytic classification of germs of conformal mappings $(\mathbb{C}, 0) \rightarrow(\mathbb{C}, 0)$, Funktsional. Anal. i Prilozhen. 15 (1981), no. 1, 1-17; English transl., Functional Anal. Appl. 15 (1981), no. 1, 1-13. MR0609790 (82h:58008)

[32] H. Whitney, Differentiable functions defined in closed sets. I, Trans. Amer. Math. Soc. 36 (1934), 369-387. MR.1501749

[33] J.-C. Yoccoz, Centralisateurs et conjugaison différentiable des difféomorphismes du cercle. Petits diviseurs en dimension 1, Astérisque No. 231 (1995), 89-242. MR.1367354 (96m:58076)

[34] T. R. Young, $C^{k}$ conjugacy of one-dimensional diffeomorphisms with periodic points, Proc. Amer. Math. Soc. 125 (1997), 1987-1995. MR.1372046(97i:58045)

[35] H. Eynard, On the centralizer of diffeomorphisms of the half-line (to appear); arXiv:0811.1173.

Mathematics Department, nui, Maynooth, Co. Kildare, Ireland

E-mail address: anthonyg.ofarrell@gmail.com

Mathematics Department, Chalmers University of Technology, and Göteborg University, SE-412 96 Göteborg, Sweden

E-mail address: maria@chalmers.se

Received 1/MAY/2009

Originally published in English 